\title{
DIE STRUKTURBODENFORMEN DER ALPEN
}

\section{Gerhard Furrer}

Die durch Temperaturerniedrigung entstandenen riesigen Eismassen des Pleistocäns beeinflußten auch das Lokalklima ihrer nächsten Umgebung. Dieses wirkte auf die Morphogenese nichtvereister, insbesondere gletschernaher Flächen, prägte ihnen charakteristische $Z$ üge auf und schuf damit einen eigenen Formenkreis. So werden beispielsweise «Strukturböden》 als Zeugen ehemaliger, morphologisch aktiver Frostperioden aufgefaßt: «Buckelwiesen》 als Bildungen der Späteiszeit, Eiskeile, kryoturbate Ablagerungen und Strukturböden s. str. Mit diesen Erscheinungen befaßt sich die Periglazialmorphologie. - Im Gegensatz zur Beeinflussung der bodennahen Luft durch Gletscher im Periglazialgebiet und den sich daraus ergebenden Formen führen wir Wirkungen des Eiszeitklimas weitab von ehemals vereisten Zonen auf primäre pleistocäne Klimaänderungen zurück (z. B. eustatische Meerspiegelschwankungen). Daher wird nicht für alle extramoränischen Spuren des Eiszeitklimas der Ausdruck periglazial verwendet.

Die vorliegende Arbeit handelt in erster Linie von Formen in den Alpen, die in der Nähe heutiger Gletscher gebildet worden sind und noch werden, oder in weiterem Rahmen von Solifluktionsformen zwischen der Vegetations- und der temporären Schneegrenze in ihrer Höchstlage. In dieser Zone - dem subnivalen Bereich - wird die Solifluktion noch nicht durch Vegetation gehemmt. Deshalb finden wir dort u. a. differenzierte Solifluktionsformen (vgl. Lit. 4). Sind einmal die Vorgänge bei der Bildung solcher rezenter Formen geklärt, dürften daraus Rückschlüsse auf fossile Periglazialformen möglich sein.

\section{MORPHOLOGISCH WIRKSAMER BODENFROST}

Wirkungen des Frostes an festem Gestein sind seit längerer Zeit untersucht worden (mechanische Verwitterung). Frostwirkungen in lockerem Gestein dagegen, besonders in feinkörnigem Verwitterungsschutt hat man erst seit der letzten Jahrhundertwende in wissenschaftliche Untersuchungen einbezogen.

Entscheidender formgestaltender Faktor im subnivalen Bereich ist periodisch auftretender Bodenfrost. Wasser und frostanfällige Böden ${ }^{1}$ sind wesentliche Voraussetzungen für die Entstehung von Frostmusterböden (s. S. 197/198). Frost - verbunden mit der Schwerkraft und der Durchtränkung der obersten Bodenschichten mit Wasser - bewirkt Erdfließen (Solifluktion).

In den Alpen hat in Bezug auf solche Bildungen speziell das Kammeis (Abb. 1) als eine Art des Bodenfrostes - wesentliche Bedeutung, während Dauergefrornis und Dauerfrostboden in den betreffenden Zonen bei uns nur ausnahmsweise vorkommen (Lit. 10) ${ }^{2}$. Das in dichten Eisnadelbüscheln auftretende Kammeis arbeitet vor allem

Abb. 1 Kammeis, Länge der Nadeln 8-10 cm. Stilfserjoch, ca. 2850 m. 5. 8. 1953, $0700 \mathrm{~h}$, Luftemperatur $-6^{\circ} \mathrm{C}$, Temperatur $2 \mathrm{~cm}$ über der Kammeisunterlage $-3^{\circ} \mathrm{C}$. - Die herumliegenden Nadeln löste ich von ihrer ungefrorenen Unterlage (Bildmitte) los. Die liegenden Büschel links im Bild lassen eine deutliche (horizontale, bzw. zur Unterlage parallele) Bänderung erkennen. Einige Nadeln sind hangabwärts gekrümmt (Bildmitte). Sogar große Steine (rechts oben) wurden vom Kammeis gehoben.

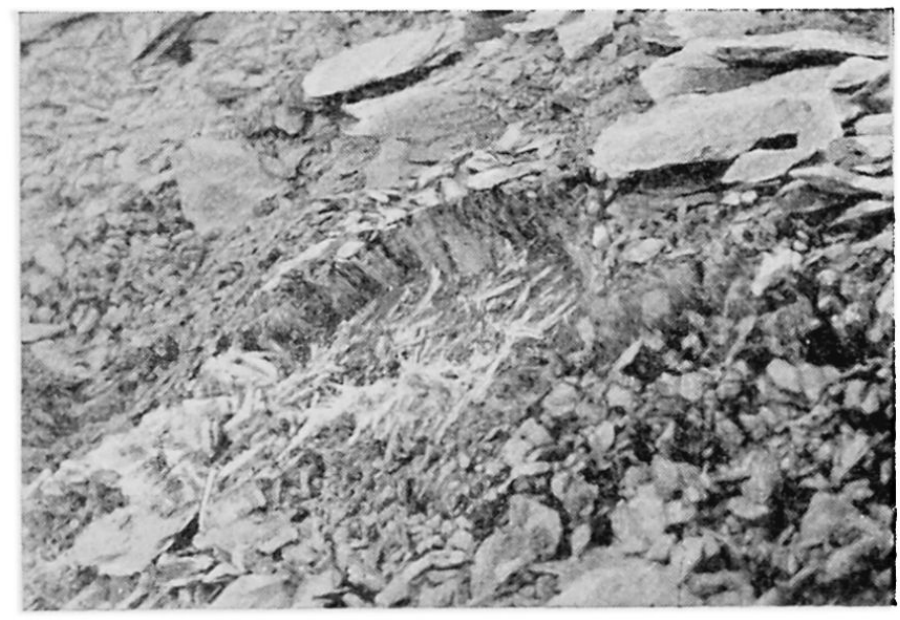

1 Die Frostgefährlichkeit eines Bodens kann durch Ermittlung seiner Korngrößenzưsammensetzung bestimmt werden.

2 In Zusammenhang mit Strukturböden ist Dauergefrornis nur von Al.Lix (Lit. 1) auf $3052 \mathrm{~m}$ in den französischen Alpen beobachtet worden. Vgl. dazu neue Beobachtungsergebnisse S. $208 \mathrm{f}$. 


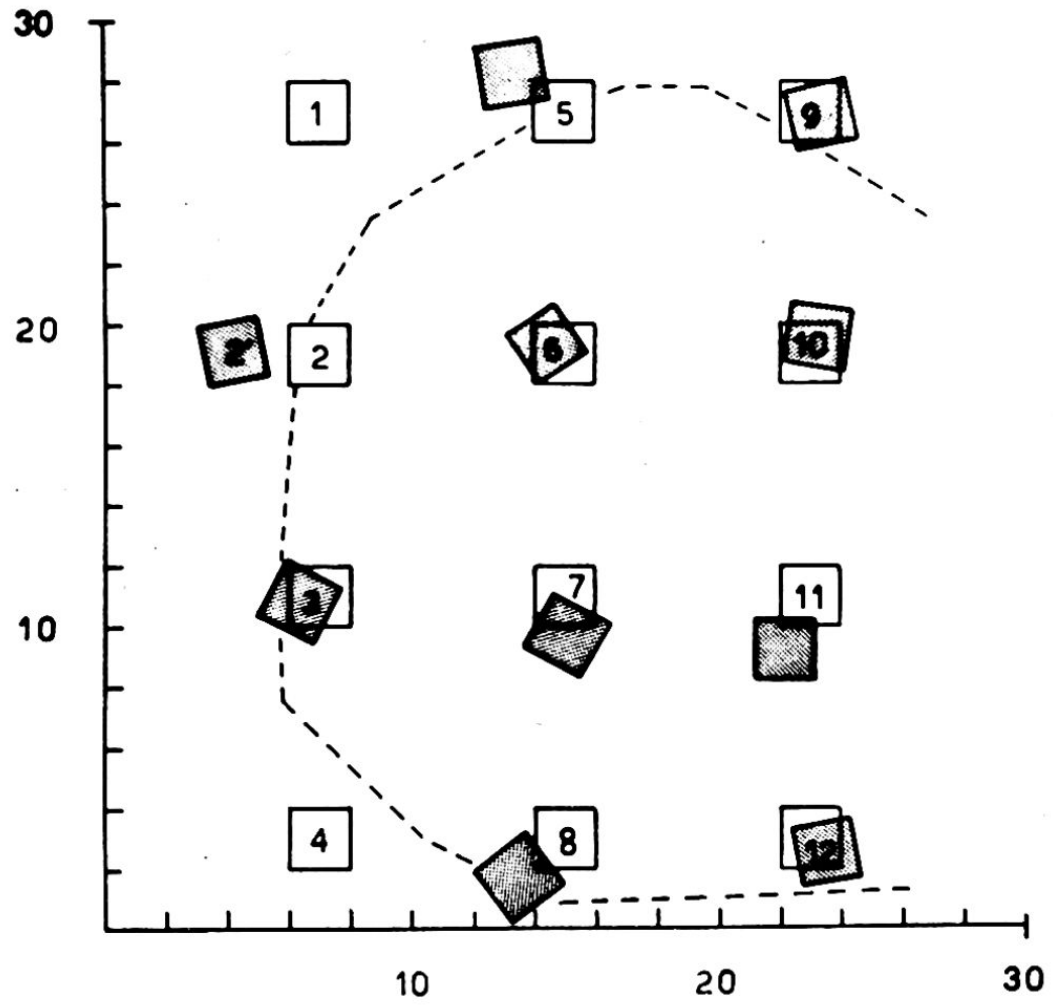

Abb. 2 Wirkung eines Kammeisbüschels während einer Nacht auf horizontaler Unterlage. Länge der Kammeisnadeln $3-5 \mathrm{~cm}$. Bewegungen festgehalten mit Hilfe von $3 \mathrm{~mm}$ mächtigen Holzplättchen (1 - 12). - Alle 12 Plättchen lagen vor der Kammeisbildung parallel zu den Koordinatenachsen. Ihre Lageveränderung wurde nach dem Abschmelzungsproze $\beta$ festgestellt (punktierte Plättchen). - Unterbrochener Strich: Grenze des Kammeisbüschels. (Glarisegg/Steckborn, $450 \mathrm{~m}, 10.12 .1952$ ). - Gehobene Partikel fallen selten in ihre ursprüngliche Lage zurück. Die Eisnadeln lösen sich oft schon zu Beginn des Abschmelzprozesses, fallen um und verfrachten das gehobene Material nach der Seite. Liegen größere Steinchen auf den Nadeln, so biegen sich besonders die rand-

ständigen büschelauswärts. Unter dem Einfluß der direkten Sonnenbestrahlung wird die Verbiegung zusehends stärker, bis die Steinchen schließlich abrutschen. Bei kleineren Büscheln verlaufen die Bewegungen analog (Entstehung von Miniatursteinringen).

mechanisch, besonders intensiv bei seiner täglichen Entstehung in den Regelationsperioden (Frühling - Frühsommer und Spätsommer - Herbst) ${ }^{3}$. Die Eisnadeln stehen

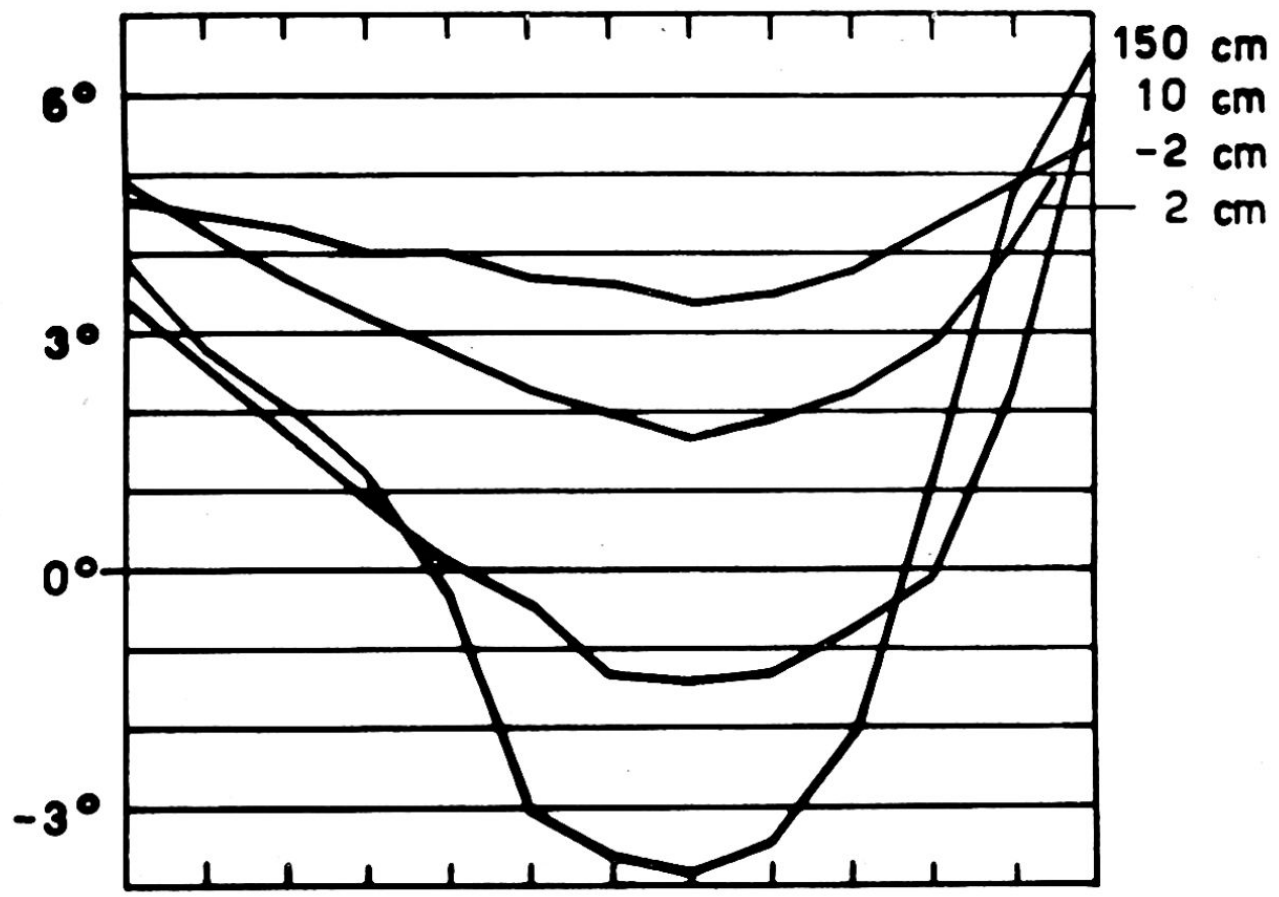

Abb. 3 Temperaraturen in verschiedener Höhe über dem Boden während einer Nacht. Keine Kammeisbildung trotz negativen Temperaturen bis in 150 und $10 \mathrm{~cm}$ Höhe über dem vegetationsfreien und feuchten Beobachtungsfeld. (20. 9. 1953, Forscherhaus im Nationalpark, $1780 \mathrm{~m})$. Auf der horizontalen Achse ist die Zeit angegeben.

2400

3 Unter Regelation verstehen wir wiederholtes Gefrieren und Auftauen. Sie erreicht für die Morphogenese höchste Bedeutung, wenn der Boden großen und häufigen Temperaturschwankungen um den Gefrierpunkt ausgesetzt ist. 
Abb. 4 Temperaturgang in der bodennahen Luft und Kammeisbildung. - Unten : Temperaturen in verschiedener Höhe über einem Kamm- 2 eisfeld (gleiches Beobachtungsfeld wie in Abb. 3). Oben: Länge der Kammeisnadeln. - $0300 \mathrm{~h}$ war das vegegationsfreie Beobachtungsfeld von einem Netz feiner Eiskristalle überzogen, eine Stunde später war die Länge der Eisnadeln meßbar. 1435 h (dicke Marke) erreichte die Sonne das Kammeisfeld. Schon eine halbe Stunde später fielen die ersten Nadeln um (21.9. 1953, beim Forscherhaus im Nationalpark, $1780 \mathrm{~m}$ ).

senkrecht auf dem Boden und tragen auf ihren Spitzen die obersten Bodenpartikel. Sie bewegen zweimal im Jahr während einer gewissen Zeit die Bodenoberfläche. - Der Abschmelzprozeß des Kammeises bringt ebenfalls oberflächliche Bodenbewegungen zustande. Beim Umfallen der Nadeln

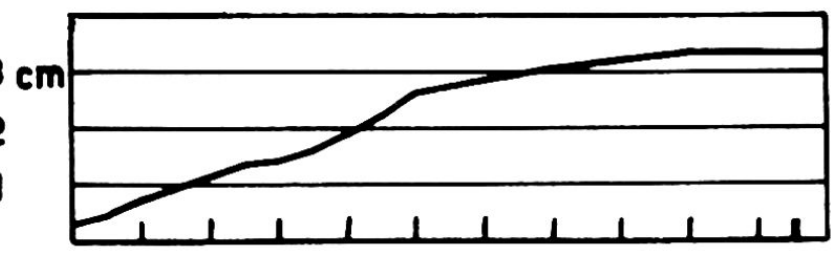
gelangen die gehobenen, «auf-

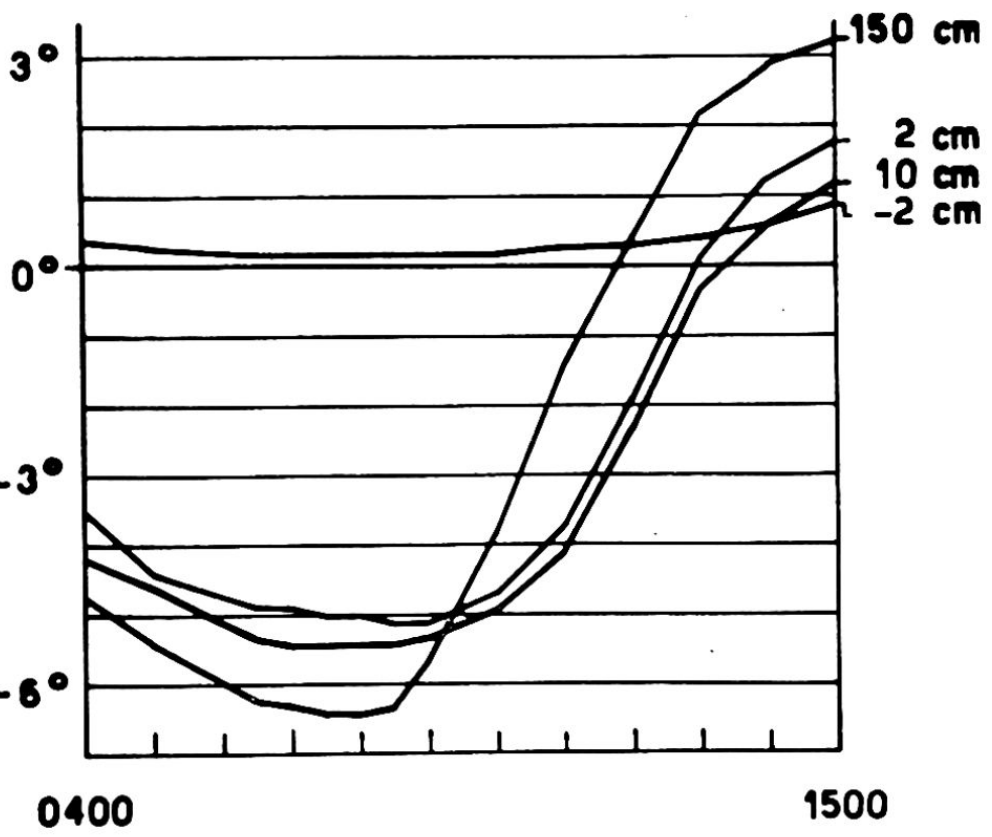
gefrorenen» Partikel nicht mehr in ihre ursprüngliche Lage zurück (vgl. Abb. 2). Auf Hängen kollern die der Frosthebung unterworfenen Steinchen - von wenigen mm Durchmesser bis zu Faustgröße - oft beträchtliche Strecken talwärts. Durch systematische Untersuchungen an Flächen von 20-25 $5^{\circ}$ Neigung ermittelte KrUmme (Lit. 7) Bewegungsbeträge der obersten Bodenteilchen von 6-8 $\mathrm{cm}$ nach jeder Abschmelzung. Bei Hangneigungen von 30-40 ${ }^{\circ}$ ist die Bewegung 2-3 mal größer ${ }^{4}$.

Freiwerdendes Schmelzwasser der Eisnadeln unterstützt diese mechanische Kammeissolifluktion: Die abgehobenen Erdteilchen und die ehemalige Kammeisunterlage werden - wenn in kurzer Zeit viel Wasser entsteht - gründlich durchtränkt und beginnen dann ruckweise zu fließen (Mikrosolifluktion auf ungefrorener Unterlage). Außerdem, weil Kammeis die obersten Partien des Bodens auflockert und darin Wasser anreichert, begünstigt es auch die Bildung von Durchtränkungsfließerde im Großen. Oft wird in kurzer Zeit genügend Wasser frei, um echte Denudation auftreten oder Racheln entstehen zu lassen.

Durch tägliche Kammeisbildung während der Nacht und das Abschmelzen am Mittag erreicht der Massentransport in einer Regelationsperiode beträchtliche Ausmaße. Beachtung verdient - insbesondere im Hinblick auf Steinringbildung - der Umstand, daß die randständigen Nadeln der Büschel häufig nach auswärts gekrümmt sind. Dies läßt sich sogar an Büscheln beobachten, die einer senkrechten Wand aufsitzen ${ }^{5}$.

${ }^{4}$ Krumme verwendete dazu $6 \mathrm{~cm}^{2}$ große und o,2 $\mathrm{cm}$ mächtige Holzplättchen. Diese «kollern» aber beim Abschmelzen und Umfallen der Eisnadeln nicht hangabwärts. Ich führte daher einige Messungen auf Hängen mit bemalten Steinen durch. Die so ermittelten Bewegungsbeträge - besonders von gerundeten Steinen - sind größer als diejenigen von KRUMME.

5 Da auch Krümmungen gegen die Schwerkraft erfolgen, z. B. bei Kammeisbüscheln auf Hängen, überhängenden Sandsteinfelsen und Grabenwänden, sehe ich im Mikroklima die für diese Krümmung verantwortliche $\mathrm{Kraft}$; vgl. dazu Lit. 4, S. 217. 


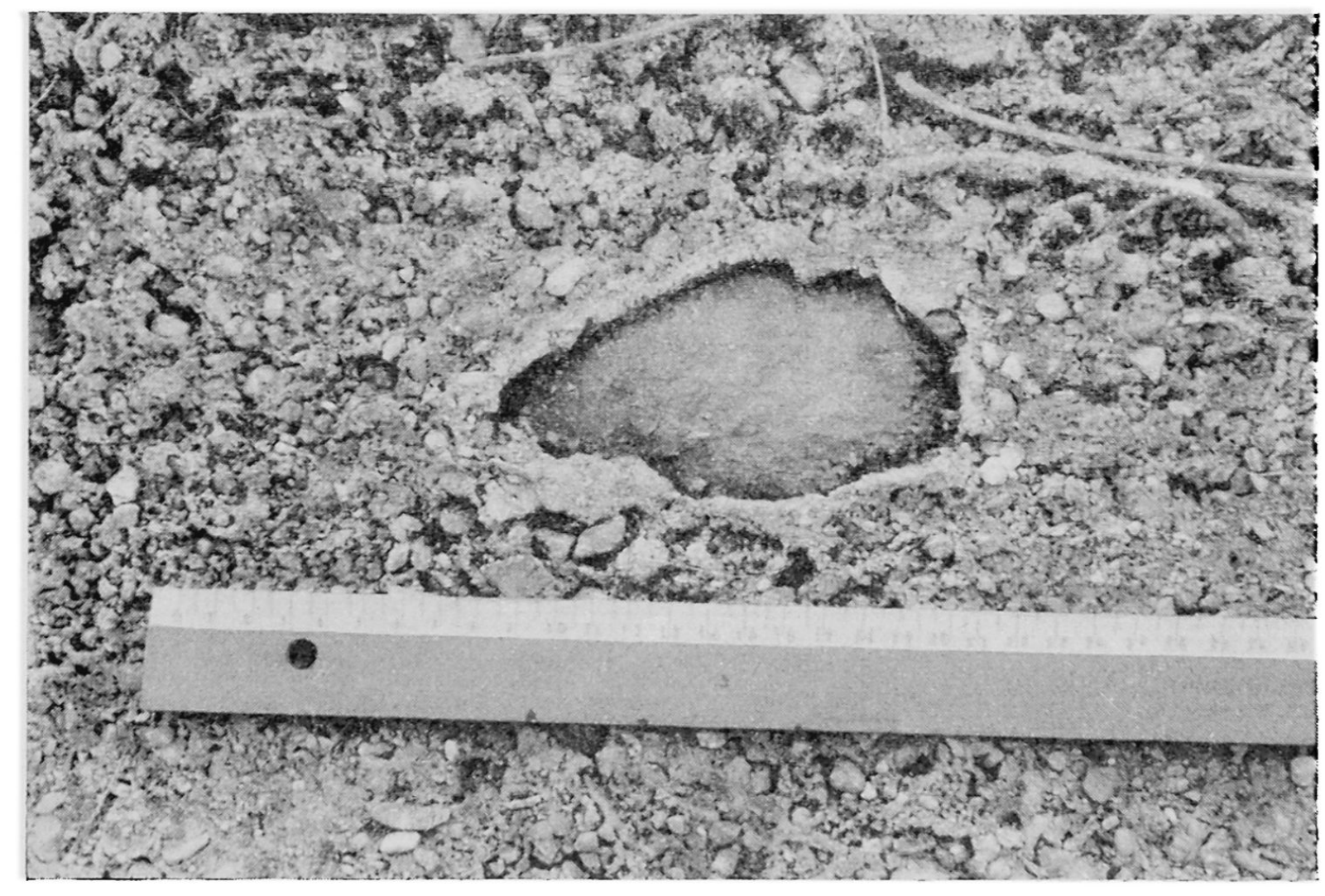

Abb. 5 Ausgefrorener Stein (vgl. dazu auch Abb. 6, Fig. B Stein 3). In den oberen Bildecken unter gehobenen Erdpartikeln Kammeis. Vom zentralen Stein strahlen Risse aus, die sich bei weiterer Hebung in den nächsten Frostnächten erweitern. Diese trennen die Feinerdeplatten des Miniaturpolygontyps von Fig. 2 in Abb. 7 voneinander. (Garten des Geographischen Institutes der Universität Zürich, 16.3.1955.)

Zur Beobachtung der Kammeisarbeit innerhalb der subnivalen Stufe sind Nordhänge oder schattige Lagen zu bevorzugen. Die tägliche Erwärmung am Mittag zerstört in solchen Fällen die Kammeisnadeln weniger häufig und die Feuchtigkeitsverluste durch Verdunstung sind kleiner. Besonders instruktive Beobachtungsergebnisse gewinnt man auf feuchten Böden: Auf sandig-tonigem Schutt und in Schneetälchen.

Außer den Lokalfaktoren (Wasservorrat des Bodens, Bodenbeschaffenheit, Vegetationsmangel) entscheiden besonders mikroklimatische Verhältnisse über das Auftreten von Kammeis. Es entsteht nur auf ungefrorenem Boden (vgl. Abb. 3 und 4). Gefriert das im Boden vorhandene Wasser, so entstehen Kräfte, welche auf die Steine des Bodens wirken. Wie stark das Mikroklima innerhalb kleiner Flächen dabei wechseln kann, veranschaulicht Abb. 5. - Liegt die $0^{0}$ Isothermenfläche hart an der Bodenoberfläche, so ist die Möglichkeit zur Kammeisbildung gegeben. Das Bodenwasser kristallisiert in Form feiner Säulchen, die bis $15 \mathrm{~cm}$ lang werden können. In der Regel bilden sich bei uns in einer Nacht höchstens 5-6 cm lange Kammeisnadeln. Schmelzen diese jedoch während des folgenden Tages nicht ab, so können sie in der nächsten Nacht wieder weiter wachsen (vgl. Lit. 4, Abb. 9). Falls die Temperatur in Bodennähe über $0^{0}$ steigt, schmelzen die Eisnadeln von oben nach unten ab. Häufig, besonders wenn Steinchen auf ihren Spitzen liegen, lösen sich die Kammeisnadeln von ihrer Unterlage und fallen der Schwerkraft folgend um.

Kammeis können wir in der ganzen Schweiz unterhalb der Schneegrenze beobachten. Die Zone der Kammeisbildung wandert vom Winter zum Spätsommer als Folge der Verlagerung seiner klimatischen Voraussetzungen vom Mittelland und vom 'Tessin bis in die höchsten schneefreien Regionen der Alpen hinauf.

Dem Kammeis verpflichtete Strukturbodenformen entstehen schon in kurzer Zeit (einige Wochen). Sie zeichnen sich durch geringen Tiefgang (Bildungen einer dün- 
Abb. 6 Ausfrieren von Steinen. Der kurze Strich rechts von jeder Figur gibt das Niveau vor dem Frosteinfluß an. Unter den gehobenen Steinen (Fig. C) feine Eisnadeln. Beim Auftauen von oben her sinken die Steine nicht in die alte Lage zurück, da die Hohlräume unter den gehobenen Steinen mit Feinerde teilweise gefüllt werden.

nen Oberflächenschicht), ausgeprägte Materialsortierung und Konstanz ihrer Dimensionen aus. Solche Miniaturformen bilden sich bei kurzperiodischem, oft tageszeitlichem Wechsel der Gefrornis - entsprechend den Strukturbodentypen der tropischen Hochgebirge.
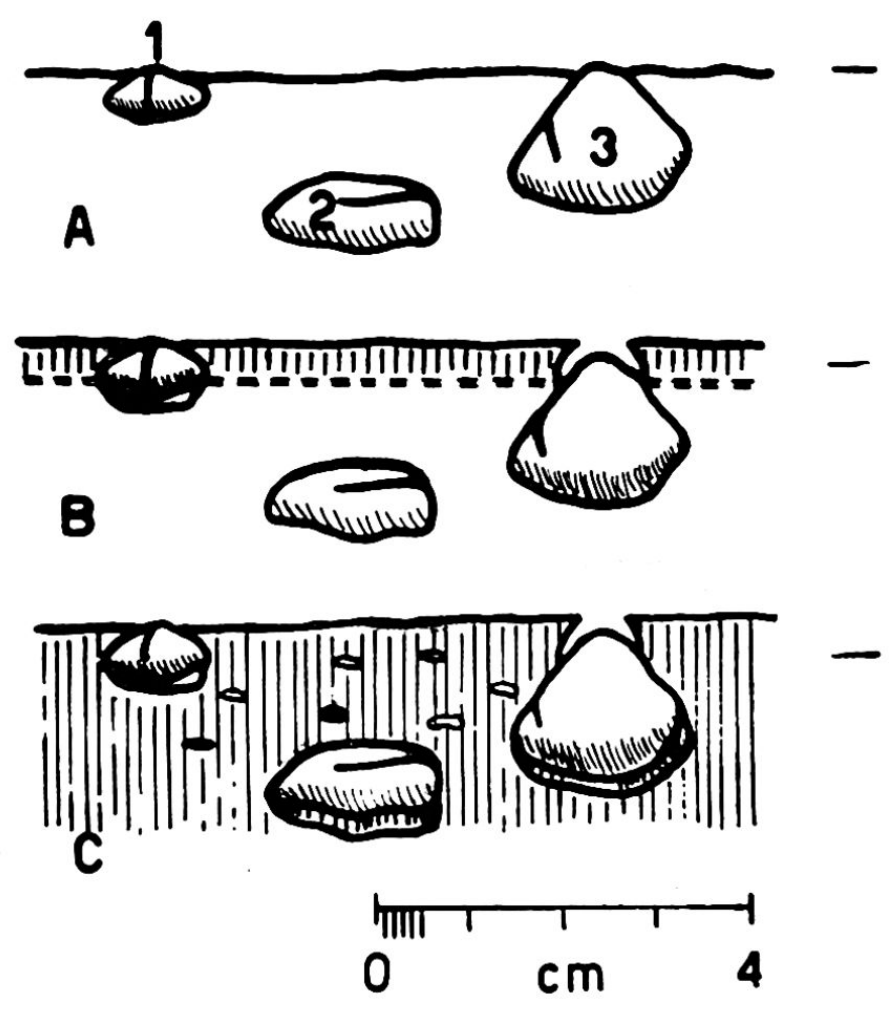

\section{WIRKUNGEN VON KURZFRISTIGEM BODENFROST}

Gefriert während einer Nacht schnee- und vegetationsfreier Boden, so entstehen häufig rings um die Steine offene Spalten (Abb. 5). Während einer Regelationsperiode können sie auf dem Boden zu unregelmäßigen Netzen erweitert werden. Die Spalten enthalten weder Wasser noch Eis. In vertikalen Profilen lassen sich dabei folgende Vorgänge beobachten: Während der Nacht ist der Frost wenige $\mathrm{cm}$ tief in den Boden eingedrungen (Abb. 6, Fig. B, schraffiert). Stein 1 ist fest mit der umgebenden Feinerde zusammengefroren und macht die allgemeine Aufwärtsbewegung des gefrorenen Bodens mit; an seiner Unterseite entsteht ein Hohlraum. In diesem sammelt sich Wasser, das - falls es gefriert - die Frosthebung des Steins beschleunigt ( Ausfrieren» von Steinen). Um Stein 3 ist eine Spalte entstanden, da er von der Aufwärtsbewegung noch nicht erfaßt wurde. Dringt der Frost weiter in den Boden vor, werden auch die Steine 2 und 3 gehoben (Fig. C). Unter ihnen beobachtet man in wenig feuchten Böden mitgehobene feine Eisnadeln von höchstens $3 \mathrm{~mm}$ Länge. - Der gleiche Vorgang transportiert auf Hängen Steine, die nahe der Oberfläche liegen, langsam hangabwärts, wobei die Bewegung eine Rinne hinterläßt.

In den Regelationsperioden frieren die obersten 3-6 cm des Bodens häufig jede Nacht ein und tauen oft jeden Vormittag wieder zu einer breiig-flüssigen Masse auf. Nach einigen Frostwechseln sondern sich unregelmäßig geformte Feinerdeplatten ab. In gefrorenem Zustand lassen sie sich als Ziegel vom ungefrorenen Unterboden abheben. Beim Zerbrechen solcher Erdziegel findet man in ihrem Innern Blasen (vgl. Abb. 6, Fig. C und Abb. 7, Fig. 2). Diese ellipsenförmigen Hohlräume liegen parallel zur Oberfläche und messen höchstens $1 \mathrm{~cm}$. Gelegentlich sind ganz feine, nadelförmige Eiskristalle oder einzelne Steinchen darin eingelagert. - Die gefrorenen, 1-3 cm mächtigen Erdziegel werden oft gehoben, falls in einer folgenden Nacht darunter Kammeis entsteht.

\section{STR UKT URBÖDEN}

Die älteste Mitteilung von Steingärtchen stammt von C. HAUsER (Jahrbuch SAC 1864, S. 159/160). - Unter Steinnetzboden verstehen wir jene Bodenoberflächen, bei denen die Steine in mehr oder weniger regelmäßiger Anordnung Feinerdebeete netzartig umschließen. Es handelt sich um eine der vielen Strukturbodenformen, bei denen Bodenskelett und Feinerde sortiert 

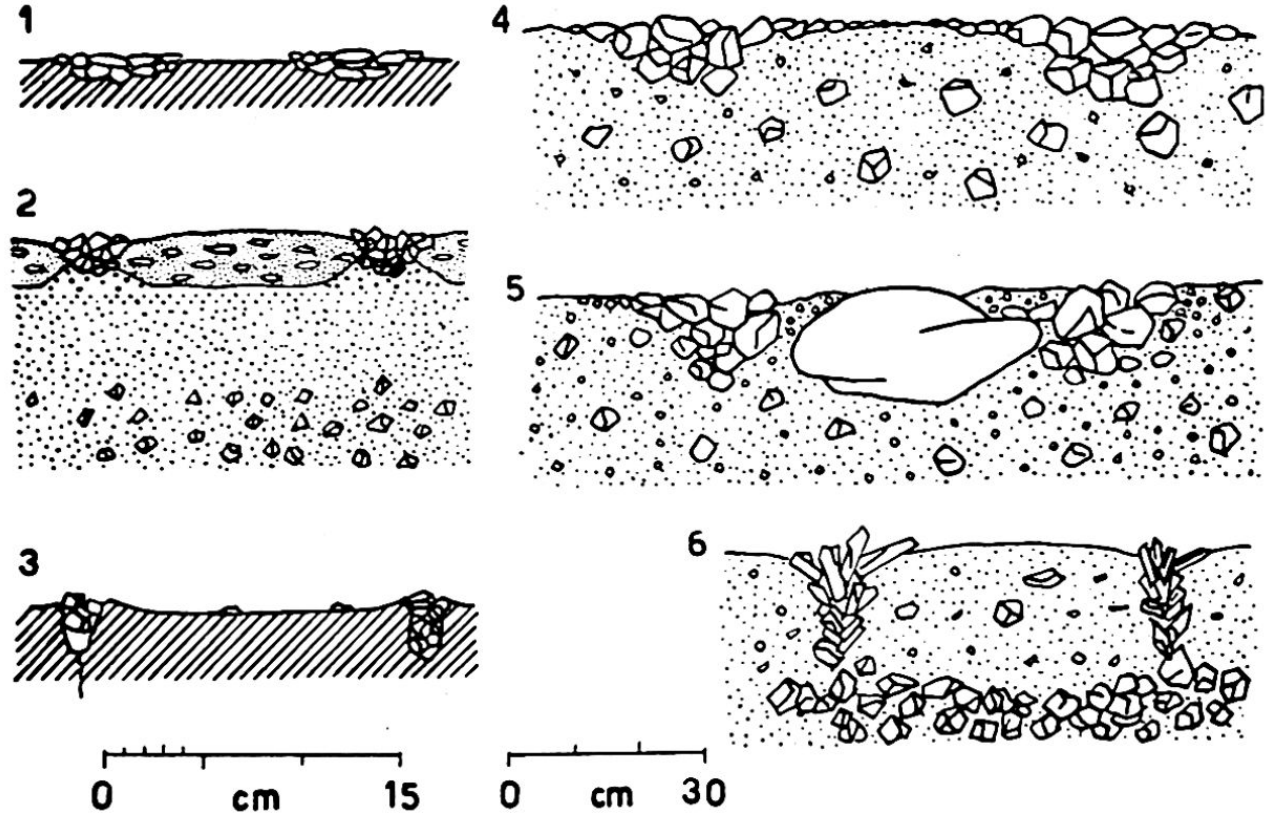

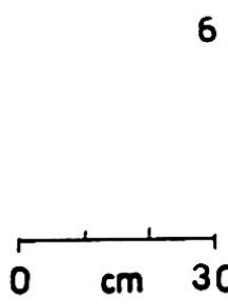

6

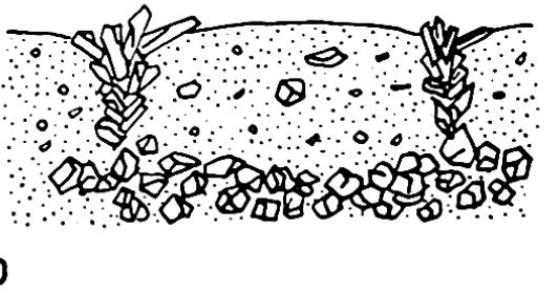

Abb. 7 Profile von Miniaturstruktur bodenformen

(links) und von Makroformen (rechts).

sind. Da Frostwirkung an der Materialsortierung maßgebend beteiligt ist, wird auch die Bezeichnung Frostgefiuge- oder Frostmusterboden verwendet.

Hie und da tritt lediglich ein einzelnes Steinpolygon meistens ein Stein. kreis - auf. Nach der Größe der einzelnen Polygone des Steinnetzbodens klassiert, unterscheide ich Miniaturund Makroformen (Durchmesser $5-25 \mathrm{~cm}$, bzw. 0,5-1,5 m).

Untersucht man die Genese dieser Formen, so lassen sich einzelne Typen erkennen. Miniatursteinpolygone beispielsweise entstehen auf 4 verschiedene Arten. Ich unterscheide daher 4 verschiedene Typen und spreche von konvergenten Bildungen. Die vorliegende Arbeit stellt somit einen Versuch dar, die Strukturbodenformen nach dem genetischen Gesichtspunkt zu klassifizieren. - Werden alle Steinnetzböden als Solifluktionsformen aufgefaßt, so stellt man sie unter dem Begriff differenzierte Solifluktionsformen (Frostbodenformen) den amorphen gegenüber (vgl. dazu Lit. 4).

Entscheidend für Strukturbodenbildung ist das Klima, i. b. das Klima der bodennächsten Luft. Die Arbeit von Winkler (Lit. 11) orientiert über die Frostverteilung in der Schweiz. Der Autor zeigt, daß «hauptsächlich die eigentlichen alpinen Landschaften die typischen Frostgebiete repräsentieren »(S. 84), und daß das Jahresmittel der Zahl der Frosttage mit der Meereshöhe steigt. - Nach SchröDER (Lit. 9) besteht in den Gebirgen eine Maximalzone der Frostwechselhäufigkeit, deren Höhe er für die Alpen im Mittel auf $1410 \mathrm{~m}$ bestimmt hat. In beckenförmigen Lagen, trokkenen Hochtälern wie im Oberengadin, erreichen die Tagesschwankungen der Temperatur ihre Höchstwerte. So weist beispielsweise Sils-Maria (1809 m) nach diesem Autor die höchste Frostwechselzahl Mitteleuropas auf (nämlich 195, S. 29).

Wir unterscheiden vom morphologischen Gesichtspunkt aus innerhalb der Frosttage zwischen Eistagen und Frostwechseltagen ${ }^{6}$. Ihre zeitliche und räumliche Verteilung wird durch Abb. 8 am Beispiel einiger Stationen veranschaulicht (Mittelwerte der Jahre 1921-1950, Terminbeobachtungen) :

1. Die Diagramme zeigen, daß Hang-/Gipfel- und Muldenlagen auseinandergehalten werden müssen.

2. Die Frostwechseltage erreichen 2 Jahresmaxima in den beiden Regelationsperioden eines Jahres. Diese nähern sich mit zunehmender Höhe gegen den Sommer und fallen über der Schneegrenze zu einem Jahresmaximum zusammen.

3. Die Zahl der Frostwechseltage ist höher, als die durch Terminbeobachtungen ermittelte, zumal im Sommer der $0730 \mathrm{~h}$ Termin nicht die tatsächliche Minimaltemperatur angibt. Weil das nächtliche Temperaturminimum mit der Zeit des Sonnenaufgangs zusammenfällt, érgeben sich gegenüber dem Termin der Morgenbeobachtung Verschiebungen bis zu 3 Stunden. Allerdings stellt SchröDER fest, daß die aus 3 täglichen Beobachtungen gewonnenen Resultate die wirksamen Schwankungen um den Gefrierpunkt gut wiedergeben.

6 Eistage: Temperatur während des ganzen Tages unter dem Gefrierpunkt.

Frostwechsel: Jeder Durchgang der Temperatur durch den Gefrierpunkt.

Frostwechseltag: Ein' Tag, an dem die Temperatur ein- oder mehrmals durch den Gefrierpunkt geht. 
Abb. 8 Eistage (untere Kurve) und Frostwechseltage (obere Kurve) verschiedener Stationen. Die obere Kurve gibt gleichzeitig das Total der Frosttage an. - Muldenlagen: Aarau - Buffalora, Hang-/Gipfellagen: Beatenberg - Säntis. (Mittelwerte der Jahre 1921 - 1950).

4. Die frühsommerliche Regelationsperiode ist die morphologisch wirksamere, weil dem Frost viel Schmelzwasser zurVerfügung steht.

5. Eistage gibt es in den Alpen auch im Hochsommer.

6. Grundsätzlich sind in den Alpen Frostbodenformen zu erwarten, die auf kurzoder langperiodischen Wechsel der Gefrornis zurückzuführen sind. Daher ist folgende Unterscheidung wesentlich :
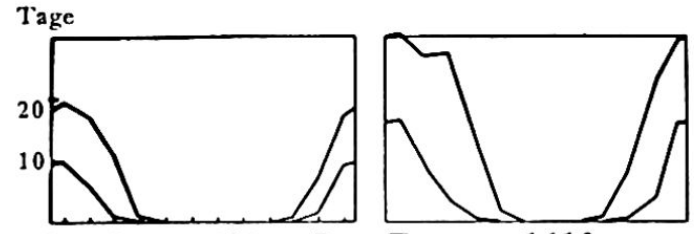

Jan. Aarau $408 \mathrm{~m}$ Dez. Zermatt $1610 \mathrm{~m}$

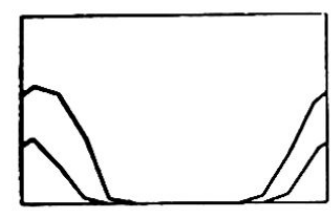

Hallau $450 \mathrm{~m}$

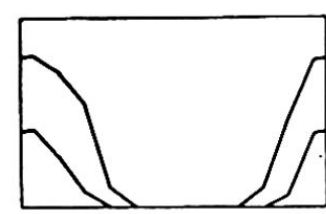

Ebnat $649 \mathrm{~m}$

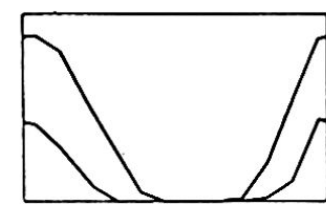

Oberiberg $1090 \mathrm{~m}$

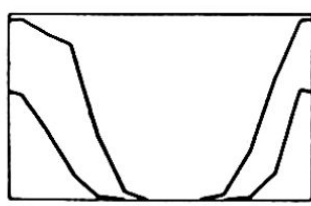

Reckingen $1332 \mathrm{~m}$

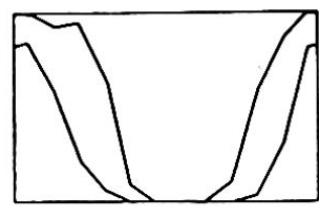

Bever $1712 \mathrm{~m}$

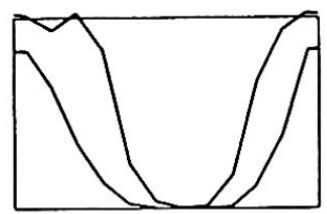

Buffalora $1977 \mathrm{~m}$

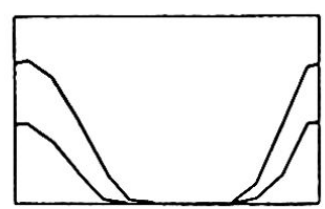

Beatenberg $1148 \mathrm{~m}$

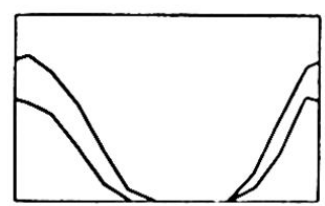

Schwäbrig $1156 \mathrm{~m}$

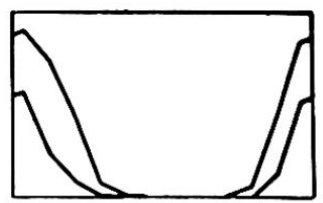

Airolo $1170 \mathrm{~m}$

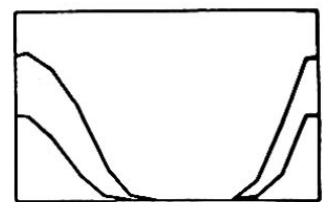

Braunwald $1280 \mathrm{~m}$

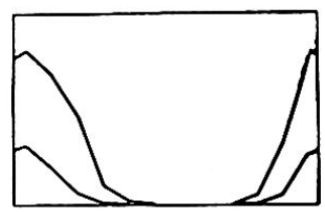

Braggio $1332 \mathrm{~m}$

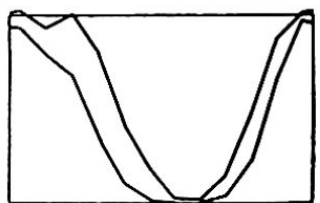

Gotthard $2095 \mathrm{~m}$

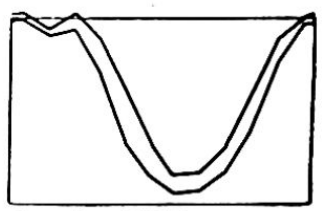

Säntis $2500 \mathrm{~m}$
Kurzperiodische Temperaturschwankungen um den Gefrierpunkt, einige Tage andauernde Fröste oder Frostwechseltage die obersten Partien des Bodens frieren häufig (jede Nacht) wenige $\mathrm{cm}$ tief ein und tauen nach kurzer Zeit wieder (jeden Vormittag) $\longrightarrow$ Miniaturfrostbodenformen von ausgeprägter Materialsortierung. länger andauernde Fröste (Wochen und Monate)
Frostbodenformen mit größeren Dimensionen und Sortierungstiefen des Materials.

[Makroformen].

Morphologisch optimal wirksam sind kurzfristige Frostwechsel nur bei fehlender Schnee- und Vegetationsdecke. Daher sind Orte mit langandauernder Schneedecke arm an Miniaturformen.

Die Schwankungen der Temperatur um den Gefrierpunkt können für die Verhältnisse an der Bodenoberfläche nicht aus den Frostwechseln der Luft erfaßt werden. Sie sind an der Bodenoberfläche und in der dem Boden unmittelbar auflagernden Luft am häufigsten. Wenige $\mathrm{cm}$ im Boden sind Frostwechseltage wegen der langsamen Ausbreitung der Temperatur viel weniger häufig als in der Luft. Dagegen ist die Zahl der Eistage schon in $20 \mathrm{~cm}$ 'Tiefe größer als in der Luft (Lit. 8, S. 140). Mit zunehmender Tiefe nimmt die Frostgeschwindigkeit ab und die Temperaturminima treten später ein als diejenigen der Luft.

\section{A. MINIATURFORMEN - POLYGONALE MUSTERUNG}

1. Steinringe als Resultat von Kammeisbildung. (Durchmesser $5-10 \mathrm{~cm}$, charakteristischer Bauplan siehe Abb. 7, Fig. 1.)

Kleine Steinringe, die durch Kammeis gebildet werden und oft zu unregelmäßigen Netzen mit relativ breiten Steinrahmen vereinigt sind, trifft man überall in den Al- 




Abb. 9 Miniatursteinnetz (Typ 3) Maßstab $1 \mathrm{~m}$. Pt. 2440, östlich Fimberpaß (10.8.55.) Die "Näpfe» sind mit Wasser gefüllt.

pen auf Lockermaterialien an. Voraussetzung für ihre Bildung sind Flächen, die lediglich eine dünne Schutt-(Stein-) decke auf feinkörniger, poröser Unterlage tragen, sowie das Vorhandensein von Wasser im Boden. In feinerdearmem Steinboden werden sie seltener ausgebildet. Meistens finden sich dann nur einzelne Steinkreise, die in ihrem Zentrum durch Kammeis aufgepreßte Erdhäufchen zeigen. - Auf dem feinkörnigen Material wachsende Kammeisbüschel heben die Steinchen - in der Regel von Haselnuß- bis Nußgröße - einige $\mathrm{cm}$ in die Höhe. Solifluktion beim Abschmelzen der Nadeln transportiert die abgehobenen Steinchen zur Peripherie der ehemaligen Büschel (vgl. Abb. 2). Dieser Vorgang wiederholt sich während einer Regelationsperiode mehrmals und ordnet die bewegten Steinchen jedesmal deutlicher in Polygonen an (vgl. Lit. 4, S. 233). Die Sechseckform ist deutlich bevorzugt, einzeln auftretende Exemplare sind annähernd kreisförmig.

Dieser Strukturbodentyp ist allgemein im subnivalen Bereich vorhanden, es scheint jedoch, daß er in der obern Zone dieser Höhenstufe besonders häufig auftritt, etwa von $2600 \mathrm{~m}$ an aufwärts. - Auf vegetationsfreien Flächen findet man einzelne Vertreter dieses Typs auch weit unterhalb der Vegetationsgrenze in den Talböden. Die Erklärung dafür liegt in der jahreszeitlichen Wanderung der Zone von Kammeisbildung.

2. Steinnetzboden entstehend durch häufigen Frostwechsel in den obersten Partien des Bodens (vgl. Abb. 7, Fig. 2 und Abb. 5 und 6).

Die durch Regelation entstehenden kompakten Erdziegel von 3-5 cm Mächtigkeit, können auch in nichtgefrorenem Zustand - falls sie beim Tauen nicht zu Fließerde werden - vom weichen und feuchten Unterboden abgehoben werden. Sie bilden in ihrer Gesamtheit auf der Bodenoberfläche unregelmäßige Muster. Ihre Grenzen sind durch Steine bestimmt, welche der Frosthebung anfänglich nicht unterlagen (vgl. Abb. 6, Fig. B, Stein 3). Mikrosolifluktion schafft aufgefrorene Steinchen von den Erdziegeln. Je häufiger die Regelation wirkt, umso schärfer wird die Trennung zwischen den Feinerdefeldern (Erdziegeln) und den Steinrahmen.

3. Steinpolygone als Folge von Nivation und Regelation (vgl. Abb. 9, Fundorte $2400-2600 \mathrm{~m}$ ).

Auf sehr stark wasserdurchtränkten Flächen, die eine relativ mächtige Steinbedeckung tragen $(20-40 \mathrm{~cm})$, findet man nach der Schneeschmelze wassergefüllte Steinnäpfe. Ihr Querschnitt ist kreisrund, außer wenn 2 benachbarte Formen zusammen- 
Abb. 10 Profil durch ein Steinpolygon. Horizontal gestrichelt: Frostboden. Gewellte Linie: Quellhorizont (s. Beschreibung S.209). Fimberpaß $(2608 \mathrm{~m})$ (10. 8. 1955).

Vgl. Detailzeichnung des Frostbodens in Abb. 13 .

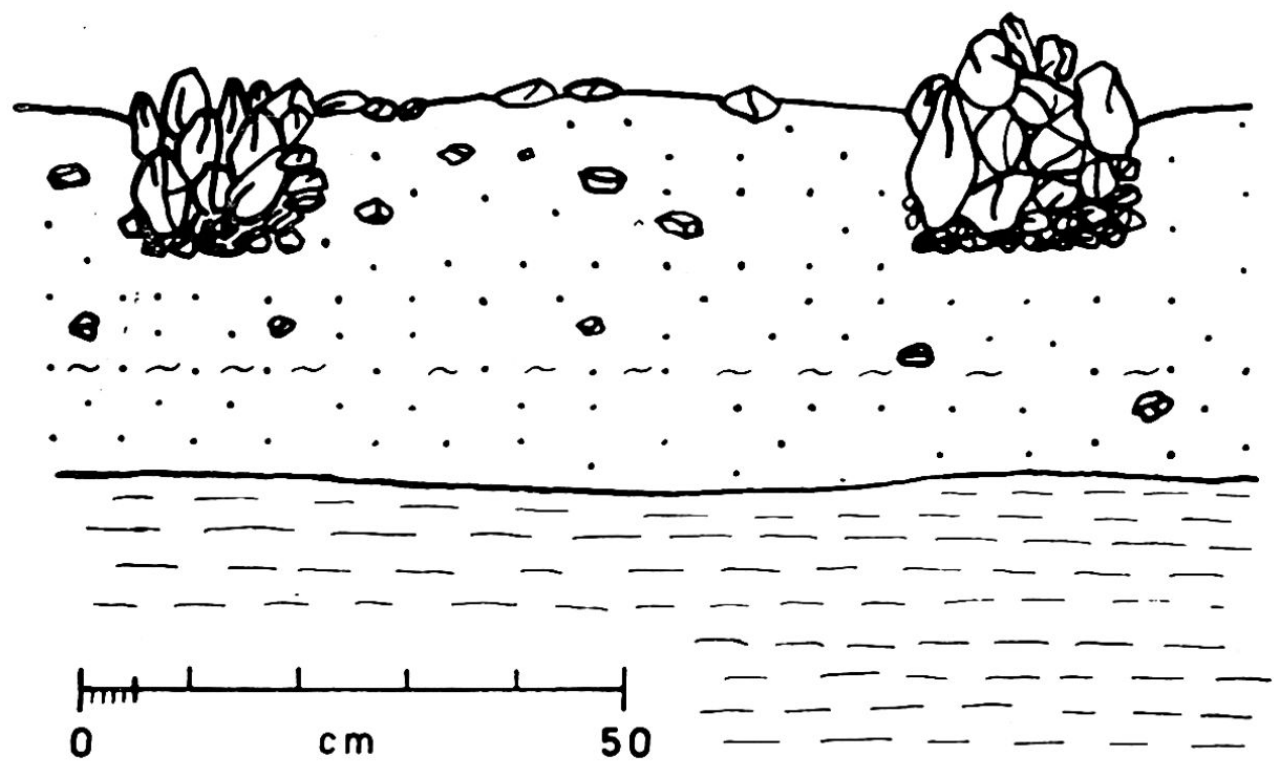

gewachsen sind (Entfernung des Zwischenwalles). Der Innendurchmesser mißt 15$25 \mathrm{~cm}$, die Napftiefe 7-15 cm. - Wenn das Wasser abgelaufen ist, sind diese Hohlformen häufig von 3-7 cm mächtigem Feinerdematerial gefüllt. Die zu Wällen zusammengescharten Steine sind oft kantengestellt, zwischen ihnen ist kein Feinmaterial eingebettet. Liegen viele Formen nebeneinander, so bilden diese Steinwälle Netze, die Flächen bis zu 1 Are überziehen. Stellenweise - besonders in der Nähe von abschmelzenden Schneeflecken - sind die Näpfe mit Schnee gefüllt. Dieser ist sehr hart gefroren, er läßt sich kaum aus der Vertiefung lösen. Die Steine der Näpfchenwand sind fest mit dem Schnee verbunden. Zwischen Schneefüllung und Steinwand befindet sich kein Feinmaterial. Durch Beobachtung von verschiedenen Entwicklungsstadien dieses Typs ergibt sich, daß dieser durch «Regelation» der Schneefüllung entsteht und die Hohlform mit der Zeit durch Feinmaterial aufgefüllt werden kann.

4. Steinnetze in Trockenrissen tonhaltiger Böden (vgl. Abb. 7, Fig. 3).

Dieser Typ entsteht durch Einspülung von Steinchen in Trockenrisse. Alle Übergänge von Trockenrißpolygonen mit keinen oder mit wenig Steinchen in den Rissen bis $\mathrm{zu}$ deutlich gezeichneten Steinnetzen können beobachtet werden. Beim Kammeistyp sind die von Steinen umrahmten Feinerdebeete annähernd kreisförmig. Dieser 4. Typ hingegen ist durch Unregelmäßigkeit der Form und der Größe der einzelnen Polygone ausgezeichnet. Ferner unterscheidet er sich von dem durch Kammeis geschaffenen Typ durch schmalere Steinrahmen $(2-4 \mathrm{~cm})$. Werden die Steinchen aus ihrer Rinne entfernt, so beobachtet man oft einen feinen vertikalen Riß als Fortsetzung der Steinrahmen nach unten.

Alle 4 Vertreter der Miniatursteinnetze entstehen auf mehr oder weniger horizontalen Flächen. Obschon es schwer hält, eine Wertung der Häufigkeit ihres Auftretens vorzunehmen, habe ich dennoch den Eindruck, daß Typ 1 und 4 ungefähr gleich häufig ausgebildet sind, der 2. und v. a. der 3. Typ etwas seltener. Bei Feldbegehungen sind der 3. und 4. am leichtesten auffindbar.

Durch Beobachtung läßt sich nachweisen, daß Mischformen dieser 4 Typen auftreten. Beispielsweise besetzen im Herbst oft Kammeisbüschel die Trockenrißpolygone und besorgen den Transport der Steinchen.

Am Beispiel der Miniaturformen ist gezeigt, daß die Betrachtung der Strukturbodenformen vom genetischen Gesichtspunkt aus verschiedene morphologische Typen erkennen läßt. Die bisherigen Untersuchungsergebnisse erlauben zwar noch keine vollständige Systematik all dieser Formen, eine solche indessen sollte angestrebt werden.

Troll (Lit. 10, S. 550) sieht nicht nur wegen dem verschiedenen Anteil des Bodeneises Schwierigkeiten für eine genetische Klassifikation der Frostböden, sondern 


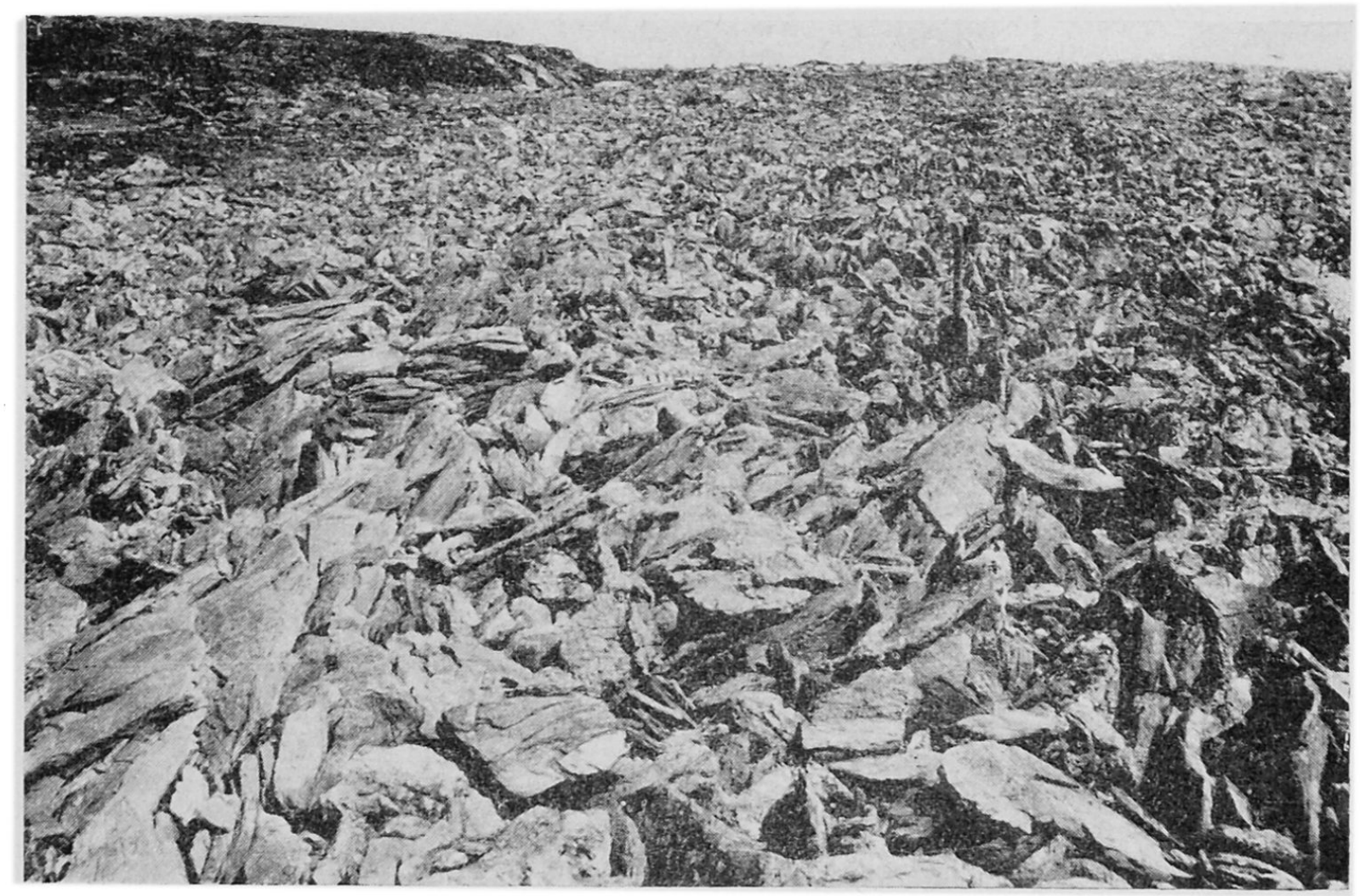

Abb. 11 Kantengestellte Steine auf stark durchnäßtem Boden. - Länge des Spatens $50 \mathrm{~cm}(816550 / 200050,2650 \mathrm{~m}, 11.8 .1955)$

vor allem weil das Bodeneis in zwei verschiedenen Richtungen wirkt: Durch Volumenvermehrung des Wassers beim Gefrieren und die daraus resultierenden Druckkräfte, sodann auch durch die Schmelzwasserbildung über gefrorenem Unterboden, was eine starke Wasserdurchtränkung des Bodens bedingt und die Verlagerungsfähigkeit der festen Bodenbestandteile vergrößert.

Die Typen 1 und 2 beweisen, wie die Art der Bodengefrornis morphologisch entscheidend ist. Festgehalten sei auch, daß für die Bildung von Miniaturformen periodischer Bodenfrost mit häufigen kurzfristigen Frostwechseln die wesentliche Voraussetzung bleibt.

\section{Steinpackungen (oder Steinrosen).}

Die Hochkantstellung von flachen, plattigen Steinen (Schiefern) trifft man außer bei Steinpolygonrahmen (vgl. Abb. 7, Fig. 6 und Abb. 10) gelegentlich auch um einen einzelnen Stein in Form eines Kranzes an. Die Schiefer sind so angeordnet, daß man den Eindruck einer seitlichen Pressung erhält (Frostschub). Der zentrale Stein als Kern kann fehlen. Steinpackungen sind feinerdearm. Sie werden auf Wechselwirkungen von Gefrieren und Tauen in wasserdurchtränktem Boden zurückgeführt. Von Steinrosen bestehen - wie ich beispielsweise auf dem Piz Beverin (2998 m) beobachten konnte - alle Übergänge zu Steinringen mit kantengestellten Steinen (Lit. 6).

Ganz selten findet man horizontale oder schwach geneigte Flächen (bis zu einer Are Ausdehnung), auf welchen sämtliche Steine - von wenigen $\mathrm{cm}$ Durchmesser bis $50 \mathrm{~cm}$ Durchmesser - in Kantenstellung angeordnet sind. Ein schönes Beispiel dieser Art fand ich am 11.8. 1955 auf Fenga Pitschna (Koord. 816550/2000050, $2650 \mathrm{~m}$ ) [Abb. 11]. Der Boden war sehr durchnäßt, stellenweise lagen die obersten Steine sogar im Wasser. Nahe dabei lagen mächtige abschmelzende Reste von Schneewehen, Eis im Boden verhinderte das Versickern des Wassers.

Die bisherige Funde lassen vorläufig keine Aussage über die Zone ihres Auftretens zu. Sie scheinen an extreme klimatische Voraussetzungen gebunden. Nach Troll 
Abb. 12 Isothermenflächen unter einem Spaltenpolygon bei kurzer Frosteinwirkung. Die Pfeile geben dieWanderungder ausfrierenden Steine an. Das Ausfrieren ist in Abb.6 dargestellt.

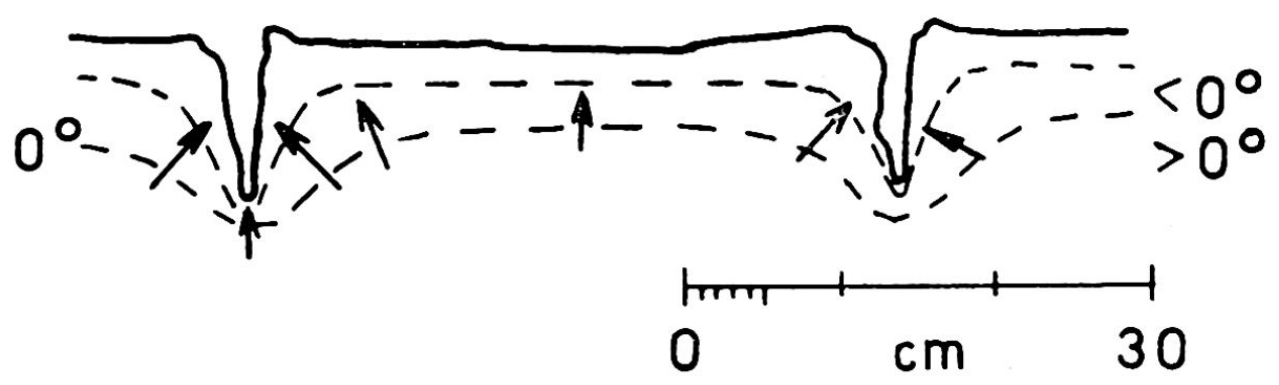

(Lit. 10, S. 599) handelt es sich um eine typisch azonale Frostbodenbildung, die weit über die klimatische Strukturbodengrenze hinausgreift.

\section{B. MINIATURFORMEN - STREIFENMUSTER}

Auf geneigten Flächen unterliegen die bisher besprochenen Formen der Hangsolifluktion (siehe Zusammenstellung der Miniaturformen). Bei Hangneigungen von $\pm 5^{\circ}$ gehen dabei die regelmäßigen Steinpolygone horizontaler Flächen in Steinellipsen über. Bei größerer Neigung als $5^{\circ}$ entstehen zur Fallinie parallele Steinstreifen. Stein- und Feinerdestreifen wechseln miteinander $a b$, oft größere Flächen bedeckend (einige Aren). Die Klassifikation dieser Oberflächenformen kann in analoger Weise wie bei den Steinnetzen vorgenommen werden:

\section{Erdstreifen.}

Auf horizontalen und schwach geneigten, vegetations- und steinfreien Flächen beobachtete ich öfters Feinerdeknospen, die durch ihre parallele Anordnung auffielen. Diese Knospen sind durch Kammeisauffrierung entstanden und die Richtung ihrer Anordnung ist durch Windwirkung zu erklären. Sie folgen ganz selten der Falllinie. Wir sprechen bei dieser Form von windgestreiftem Auffrierboden. Die einzelnen Streifen messen Breiten von 2-4 cm. Meistens fehlen Kammeisnadeln in den Zwischenräumen der Erdkrümel.

Nach meinen bisherigen Beobachtungen bringen windgestreifte Auffrierböden keine Strukturböden hervor. Ausgenommen sind jene Fälle, wo die Feinerdeknospen der Hangsolifluktion unterliegen. Bei bestimmten Hangneigungen läßt sich feststellen, daß die Kammeisauffrierungen (Erdknospen) nach der Fallinie orientiert sind. Die oberflächlich aufliegenden Steinchen werden seitlich der Erdknospenreihen zu Streifen zusammengeschoben: Es entstehen zur Fallinie parallele Stein- und Erdstreifen von durchschnittlich je $5 \mathrm{~cm}$ Breite. Da in diesen Streifen die Erdknospen das Primäre sind, wird dieser Typ als Erdstreifenboden bezeichnet.

Ich hatte verschiedentlich Gelegenheit, Übergänge von Steinnetzen zu Erdstreifen zu beobachten (Lit. 4 und 6). Auf horizontaler Fläche findet man Feinerdeknospen, welche die Schuttdecke beulenartig durchdringen; die Steinchen liegen kreisförmig darum herum. Bei beginnender Hangneigung sind die einzelnen Steinringe nicht mehr in einem mehr oder weniger regelmäßigen Netz, sondern in kurzen Reihen angeordnet. Verfolgt man dieselben einige Dezimeter weit talwärts, so treten anstelle von einzelnen Steinringen kurze geschlossene Feinerdestreifen als trennende Wälle der «Steinstreifen» auf. Weiter hangabwärts - falls die oberflächliche Steinbedeckung nicht zu mächtig wird - findet man in den Erdstreifen immer weniger unterbrechende Steinkommissuren.

Am Morgen, an schattigen Hängen auch am Nachmittag, stehen noch Kammeisnadeln in den Erdstreifen, hauptsächlich nach der Schneeschmelze. Die Erdstreifen sind in diesem Zustand aufgequollen und überragen das Niveau ihrer benachbarten «Steinstreifen». Einige Zeit der Sonne ausgesetzt, sinken sie wieder in sich zusammen. Allfällige Steinchen, die noch auf den Erdstreifen liegen, werden beim Auffrieren 


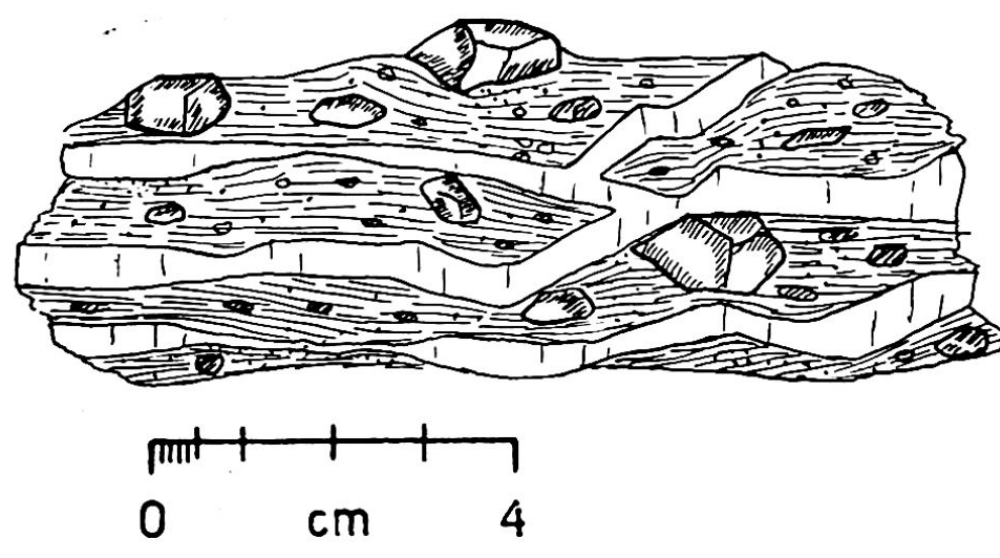

Abb. 13 Handstück aus dem Frostboden von Abb. 10. - Weiße Bänder : Eisplatten.

mitgehoben und gleiten beim Abschmelzen oder schon vorher seitlich zu den Steinrahmen ab.

Die Erdstreifen folgen der Fallinie, größeren Hindernissen - Steine oder Vegetationspolster - weichen sie durch stromlinienartiges Umfließen aus. Sie scharen sich dabei und streben unterhalb solcher Stellen wieder auseinander.

\section{Steinstreifen (s. str.)}

Durch Deformation von Steinnetzen entstehende Steinstreifen sind unter den Miniaturformen stark vertreten. Für die Deformation ist die Hangsolifluktion verantwortlich. Wissenschaftlich besonders wertvoll sind die Übergangsformen von horizontalen zu geneigten Flächen. Deformierte Steinpolygone und entlang der Falllinie ausgezogene Steinellipsen treten schon bei schwachen Neigungen auf. - Im Gegensatz zu den Erdstreifen variieren die Dimensionen dieser Typen beträchtlich; die Flächen zwischen den Steinstreifen können breit $(15-20 \mathrm{~cm})$ oder schmal $(10 \mathrm{~cm})$ sein. Wie beim Steinnetzwerk treten Formen auf, bei denen die Steinchen in vorgebildete Rinnen - Trockenrisse, Regenracheln, kleine Schmelzwasserrinnen - nachträglich eingeschwemmt wurden. Es gibt auch hier Formen, die durch Zusammen-

\section{ZUSAMMENSTELLUNG DER MINIATURFORMEN}

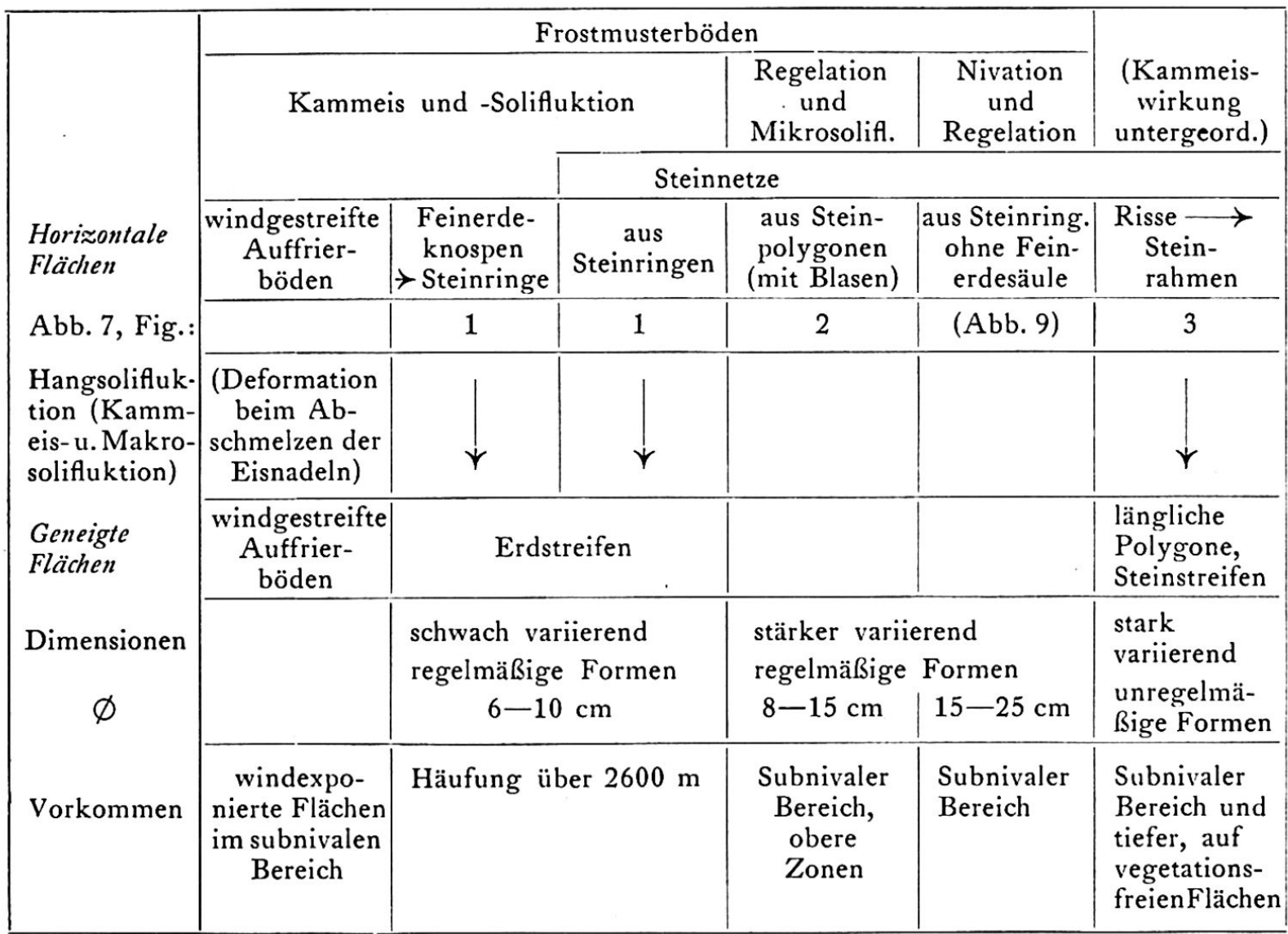




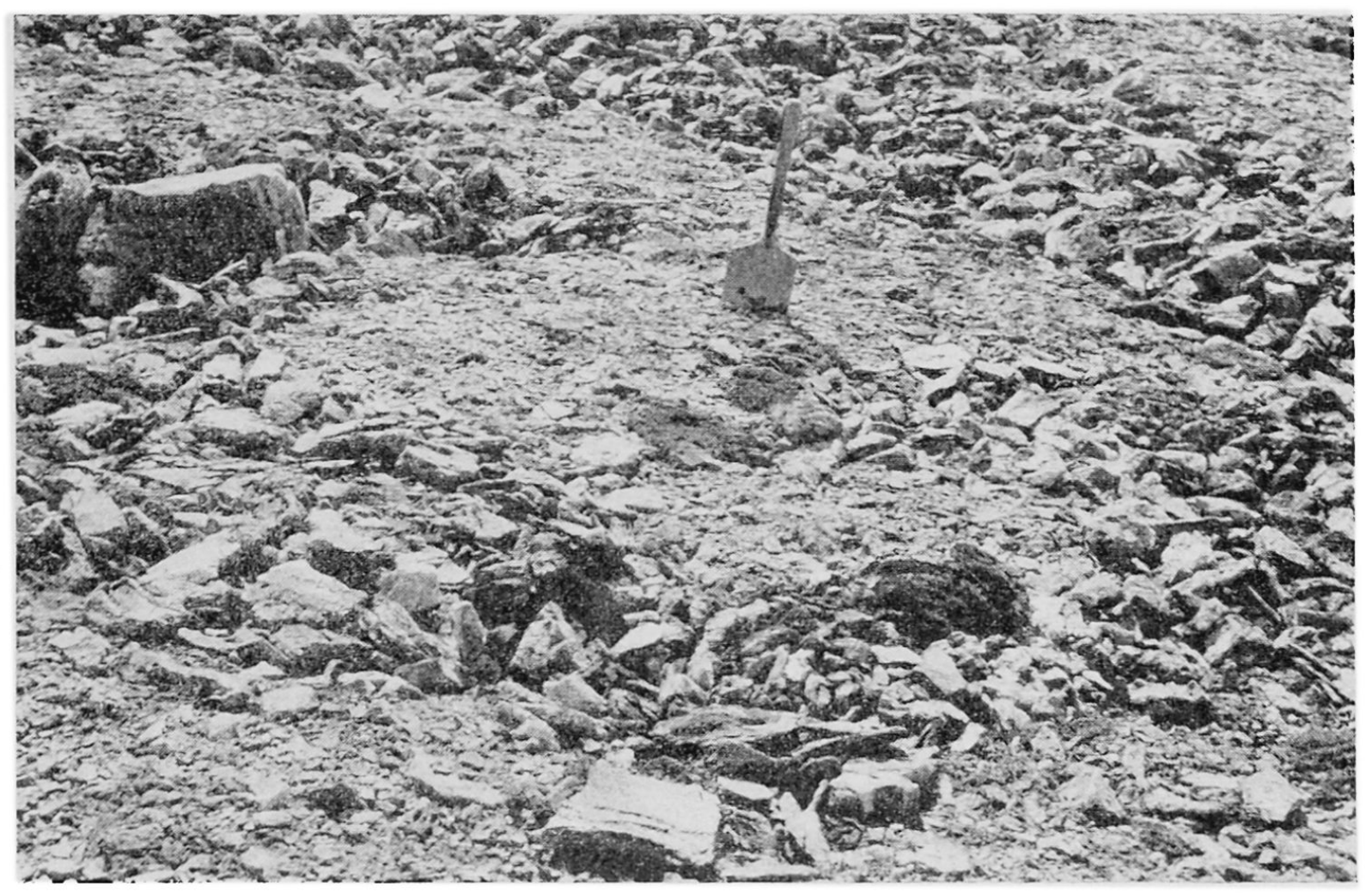

Abb. 14 Deformiertes Steinpolygon. Fallinie: Spaten-rechte untere Bildecke. - Kleinfimber, Koord. 816580/199100, $2660 \mathrm{~m}$ (11. 8. 1955). (Länge des Spatens $50 \mathrm{~cm}$. )

wirken verschiedener Ursachen entstehen: z. B. Erweiterung von Trockenrissen durch Schmelzwasser, Kammeissolifluktion, welche die Steinchen zu den Regenracheln transportiert.

\section{KONVERGENTE UND HOMOLOGE FORMEN}

Ihrem Aussehen nach gleiche Erscheinungen können durch verschiedene Kräfte entstanden sein (konvergente Bildungen). Als eigentliche Frostmusterböden gelten nur jene Typen, die durch den Bodenfrost geschaffen wurden, z. B. Steinringe und Erdstreifen durch Kammeis. Bei den andern Formen kann Kammeis teilweise mitgewirkt haben.

Homologe Formen werden durch genetische Betrachtung erkannt, z. B. Erd- und Steinstreifen, die aus Steinnetzwerk entstehen (vgl. dazu die Zusammenstellung der Miniaturformen).

Die Kleinform der Frostmusterböden in den Alpen entstehen durch Kammeis und Regelation in kurzperiodischem, oft tageszeitlichem Rhythmus der Bodengefrornis. Sie sind den Frostbodenformen der tropischen Hochgebirge vergleichbar. - Im Folgenden soll gezeigt werden, daß größere Formen - dem andern Grundtyp der Bodengefrornis, dem subpolaren vergleichbar - in den untern Zonen des subnivalen Bereiches der Alpen auftreten. Die Namen «tropische» und «polare» Bodenformen sollten aber für die Solifluktionsformen der Alpen nicht verwendet werden, da es sich bei unseren Frostmusterböden um Übergangs- und Mischformen der beiden Grundtypen handelt.

C. POLYGONE GRÖSSERER DIMENSIONEN (Makroformen) Abb. 15 und 16)

Auf jungen Moränen im Vorfeld heutiger Gletscher treten gelegentlich Steinringe von größeren Durchmessern und Sortierungstiefen als denjenigen der Miniaturformen auf. Dadurch wird gezeigt, daß nicht alle Steinpolygone in unseren Alpen durch Kammeiswirkung und kurzfristige Frostwechsel zu erklären sind, sowie daß auch heute noch Großformen von Strukturbodentypen bei uns gebildet werden. Es müssen noch 


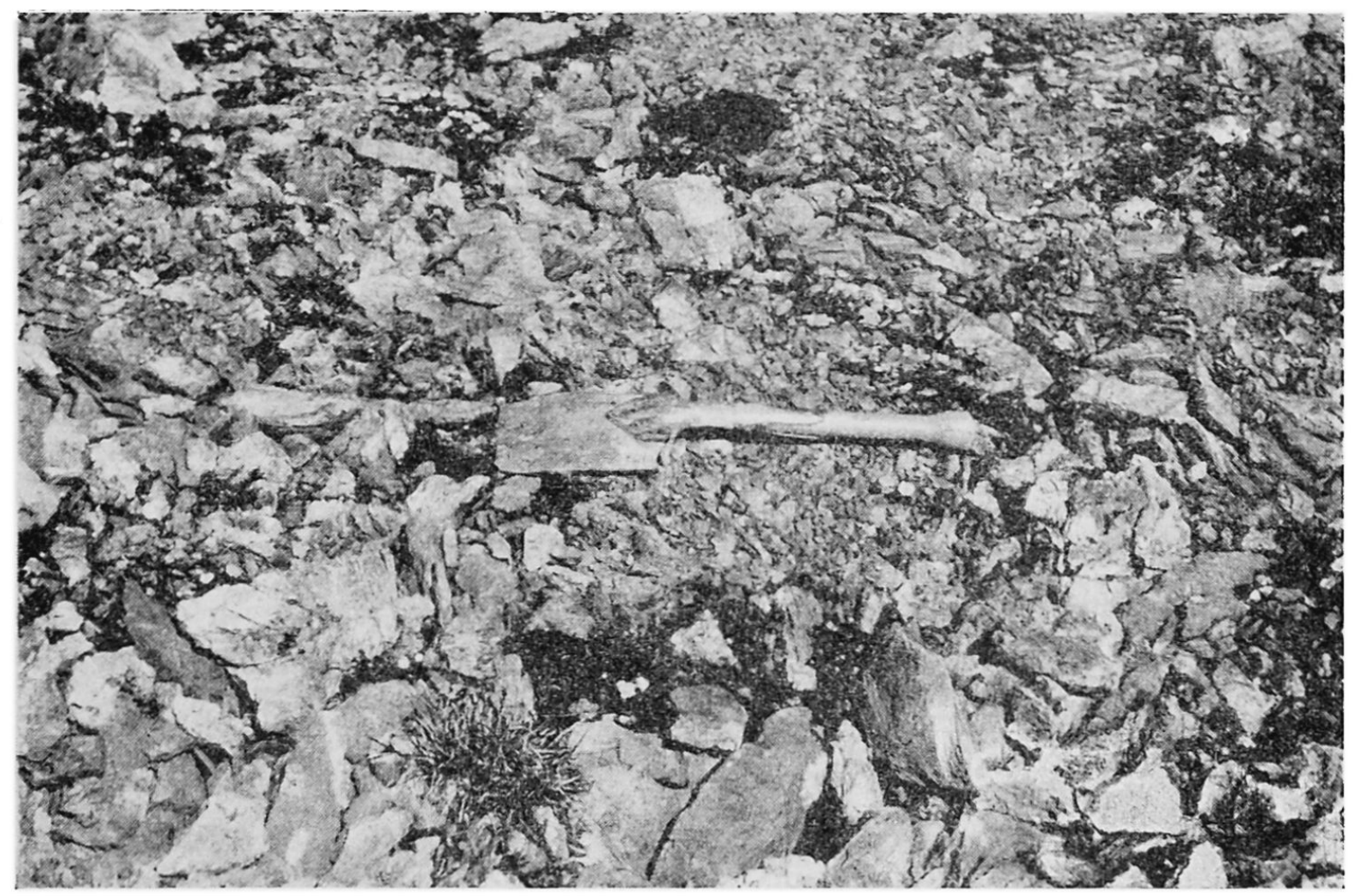

Abb. 15 Steinring auf einer Verflachung unterhalb der Streifen von Abb. 18. (11.8. 1955.)

andere Vorgänge als die bisher beschriebenen wirken - wie wollte man sonst die Steinpackungen oder jene seltenen Steinringe erklären, die in ihrer Feinerdesäule einen über kopfgroßen Stein als Kern enthalten? (vgl. Abb. 7, Fig. 5 und Lit. 5, Abb. 2).

1. Steinringe vor der Zunge des Morteratschgletschers (vgl. Abb. 7, Fig. 4 und 5)

Der Ort, wo ich am 3.8. 1953 Steinringe und Steinnetzboden beobachtete, befindet sich unweit der Gletscherstirne, innerhalb des Gletscherstandes von 1878, auf einigen flachen Stellen mit feinkörnigem Schutt inmitten von Schotter und groben Blöcken der jüngsten Moränen des Morteratschgletschers. Die einzelnen Polygone der Steinnetze waren i. a. sechseckig, auf schwach geneigter Unterlage länglich-ellipsenförmig. Die Diagonalen dieser Formen maßen $40-70 \mathrm{~cm}$, die Durchmesser der zentralen Feinerdezylinder an der Oberfläche $30-40 \mathrm{~cm}$; die Steinrahmenbreite schwankte zwischen 15 und $30 \mathrm{~cm}$, die durchschnittliche Tiefe der Steinrahmen betrug $15 \mathrm{~cm}$, und ihre Steine erreichten Faustgröße. Die Materialsortierung - die Scheidung nach der Korngröße - ließ sich nur $10 \mathrm{~cm}$ tief feststellen. Weiter unten waren die Steine regellos im Feinerdematerial eingebettet. An der Oberfläche bot sich das gleiche Bild wie bei Kammeis-Steinringen: Die größten Steine lagen im mittleren Teil der Steinrahmen, während gegen das Zentrum der Feinerdesäule hin die Größe der Steine allmählich abnahm (vgl. Abb. 7, Fig. 4 und 5).

Mitten unter diesen Polygonen fand ich eines, dessen Feinerdezentrum einen groBen Stein als Kern enthielt (Abb. 7, Fig. 5). Auch KINZL ${ }^{7}$ bemerkte unter Feinerdefeldern von Steinringen in Tiefen bis $30 \mathrm{~cm}$ große Steine. Materialsortierung und Aufwölbung der Polygonzentren sind bei solchen Formen besonders ausgeprägt.

Troll (Lit. 10) führt diese Beobachtungen auf die stärkere Frosthebung größerer Blöcke zurück (Korndurchmesser über $20 \mathrm{~cm}$ ). - Kurzfristige Fröste dringen nicht so tief in den Boden, als daß sie unter den Blöcken Eis zur Frosthebung bilden könnten. Daher belegen diese Sonderfälle die Wirkung langandauernder Fröste. Stein-

7 Beobachtungen über Strukturböden in den Ostalpen. - Petermanns Geogr. Mitt. 74, 1928. 
Abb. 16 Steinringe auf Kleinfimber (in derNähe der kantengestellten Steine von Abb. 11).

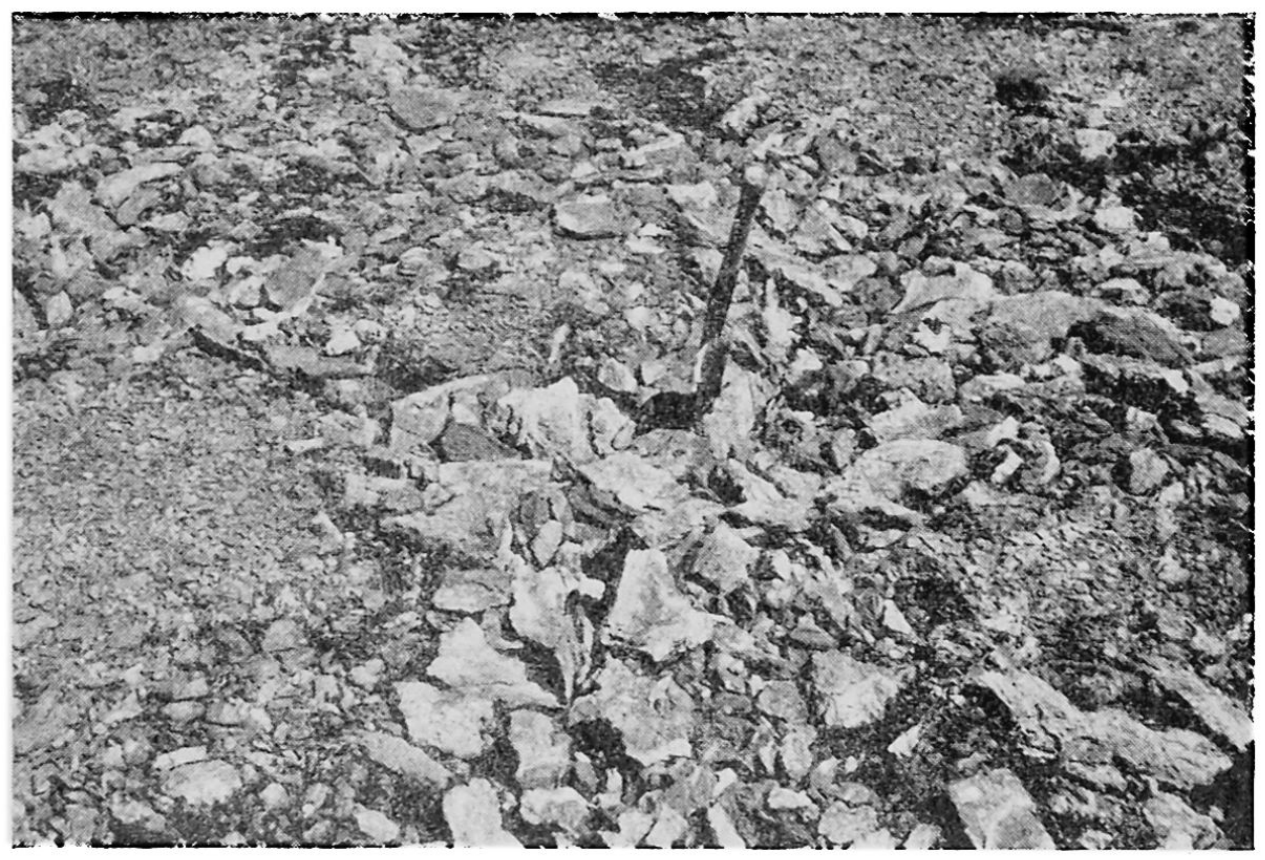

kerne sind keine Voraussetzung für Polygonbildung, sie wirken aber als aklimatische Verstärkung bei der Genese mit.

\section{Steinpolygone mit sohlenständigen Steinrahmen (Abb. 7, Fig. 6)}

Der Steinrahmen des in Fig. 6 dargestellten Polygons reicht bis zu einer Steinsohle hinab. Ein vertikal angelegtes Profil unterscheidet sich von jenem der napfförmigen Miniaturform (Typ 3, Abb. 9) durch das Vorhandensein von Steinen in der Feinerdesäule und durch die besondere Form der Steinrahmen: Bei den wenigen mir bekannten sohlenständigen Makroformen verjüngen sich die Steinrahmen von oben nach unten. Schieferförmige Steine sind im ganzen Rahmen kantengestellt. Etwas Feinkies (0,2-2 cm Korndurchmesser) und einige wenige größere Steine finden sich regellos in der Feinerdesäule verteilt. Während der Schneeschmelze zirkuliert in den Steinrahmen viel Wasser, das dieselben vertieft.

Besonders bei sohlenständigen Steinpolygonen bemerkte ich, wie ihre Bildung von feinerdereichen Stellen im Verwitterungsschutt ausgeht. Entsprechend der ungleichen Verteilung von Bodenskelett und Feinerde erfolgt differenzierte Frosthebung und Aufwölbung der Oberfläche.

\section{Steinrahmen in Spalten}

Viele Steinnetze sind auf Spaltennetze ausgetrockneter Fließerde zurückzuführen (vgl. dazu auch Miniaturtyp 4). Diese Entwicklung kann beim Studium von verschiedenen genetischen Stadien gut verfolgt werden. Wie bei den Miniaturformen werden die Risse oberflächlich mit Steinen gefüllt, v. a. aber bewirken sie auch differenzierte Frosthebung und differenziertes Ausfrieren von Steinen (vgl. Abb. 12 und 6).

Messungen von Bodentemperaturen ergeben, daß die Kälte auch von den Spalten aus in den Boden eindringt. Die gefrierende Erde dehnt sich nach oben und seitwärts zu den Spalten. Die Wanderung der Steine erfolgt somit nach oben und gegen die Spaltenränder zu (vgl. Abb. 12). Diese seitwärts gerichtete Materialsortierung dauert auch bei gefüllten Spalten (Steinrahmen) an, da ihr loses Gefüge den Verlauf der Isothermenflächen nicht wesentlich zu ändern vermag. - In Profilen durch Feinerdesäulen von Übergangsformen Spaltennetz-Steinnetz stellt man oft gegen die Rahmen eine größere Häufigkeit der Steine fest. 




Abb.17 Steinstreifen über Frostboden. - Die Feinerde ist stark mit

Wasser durchtränkt. In der Bildmitte der Abdruck eines Schuhs, das entstandene Loch füllte sich sogleich mit Wasser. Rechts desSpatens kanten. gestellte Steine. (Kleinfimber, ca. $20 \mathrm{~m}$ tiefer als Abb. 11 aufgenommen, 11.8 . 1955.)

\section{Steinpolygone mit aufgewölbten Feinerdezentren}

Frostbeulen weisen in ihrem Innern mehr oder weniger waagrecht liegende Eislinsen auf. Das Eis ist rein und klar. Die Mächtigkeit der Linsen und ihr vertikaler Abstand schwanken zwischen wenigen Millimetern und $2-3 \mathrm{~cm}$. Nach RUckLI (Lit. 8, S. 15) sind im Untergrund eines Alpenflugplatzes schon Eislinsen von 20 und mehr cm Mächtigkeit beobachtet worden. - Beim Gefrieren von Böden mit genügend Kapillarität über nichtgefrorenem Unterboden wird Wasser in die Gefrierzone gesogen. Allerdings muß zu Beginn der Frostwirkung der Boden bereits kapillar durchfeuchtet sein. Die Aufwölbung rührt vom hochgesogenen Wasser her. Frostbeulen als Feinerdezentren von Steinpolygonen bis zu $3 \mathrm{~m}$ Durchmesser und von kantengestellten Steinen tragen als Eigentümlichkeit gelegentlich sekundäre Miniatursteinpolygone.

\section{Steinnetzboden über Frostboden (Abb. 15 und 16)}

Am 10. und 11. August 1955 untersuchte ich im Fimbergebiet (N Schuls) außerordentlich instruktive Strukturbodenformen. In der vorangegangenen Woche herrschte schlechtes Wetter, am 8. und 9. August fiel Schnee bis auf $2600 \mathrm{~m}$. In nordexponierten Lagen, Karen, Nischen und unterhalb der Pässe (Cuolmen d'Fenga, $2608 \mathrm{~m}$ und Fenga Pitschna, $2650 \mathrm{~m}$ ) lagen beträchtliche Reste von Schneewehen des letzten Winters. Schon 1954 schmolzen ebenda die Schneemassen über ca. $2400 \mathrm{~m}$ nicht gänzlich ab. Die Monatsmitteltemperaturen - berechnet auf Grund der Messungen von Säntis (2 500 m, 1901-1940) und Zugspitze (2962 m, 1901-1949) - betragen für das Gebiet des Fimberpasses $(2600 \mathrm{~m})$ folgende Werte $\left(\mathrm{C}^{\circ}\right)$ :

$\begin{array}{lrlrlr}\text { Januar } & -9,0 & \text { Mai } & -0,4 & \text { September } & 2,3 \\ \text { Februar } & -9,3 & \text { Juni } & +2,5 & \text { Oktober } & -1,3 \\ \text { März } & -7,7 & \text { Juli } & 4,4 & \text { November } & -5,3 \\ \text { April } & -5,1 & \text { August } & 4,4 & \text { Dezember } & -7,9\end{array}$

Jahr $-2,7$

In Höhen von 2500-2 $700 \mathrm{~m}$ waren große horizontale oder schwach geneigte Flächen des Bodens außerordentlich stark mit Schmelzwasser durchtränkt. Fließerde überzog ganze Hektaren, stellenweise lag auf ihr sogar Wasser. Das Gehen war sehr mühsam, überall sank man in dieser «breiigen» Masse bis an die Knöchel ein. Zum Vorwärtskommen benützten wir größere Steine oder - wie die Grenzwachtsoldaten Steinrahmen von Strukturbodenformen. Wo reichlich Steinschutt lag, quoll beim Daraufstehen oft Wasser an die Oberfläche. Dabei hörte man das «Gurgeln» des 
Abb.18 Steinstreifen. - Hangneigung $8-12 \circ$.

$(816500 / 200050$, $2660 \mathrm{~m}$,

11. 8. 1955.)

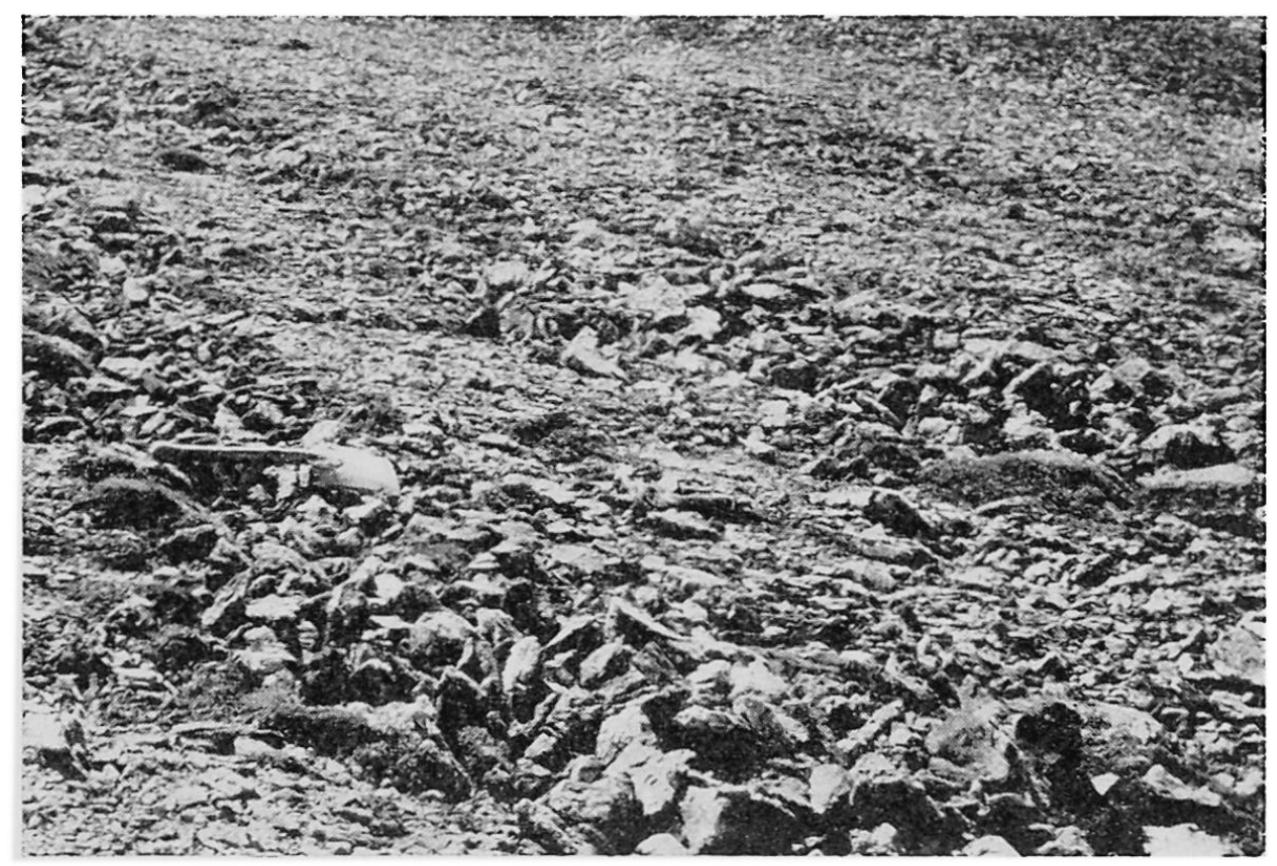

Wassers zwischen den Steinen. Große, deutlich ausgebildete Strukturbodenformen zierten diese Flächen; Steinnetze überzogen Felder von mehreren Aren Ausdehnung.

Das Graben mit dem Spaten war schwierig, da in jedes ausgehobene Loch sogleich wieder Fließerde strömte. Hatte man endlich eine kleine Grube offen, so füllte sie sich mit Wasser. Einmal gelang es mir, in einer tonhaltigen Fließerdesäule eines Steinpolygons ein Profil bis in $35 \mathrm{~cm}$ Tiefe sauber abzustechen (wenige $\mathrm{m}$ östlich Pt. 2 608, Abb. 10). 28-30 cm unter der Oberfläche bildete sich sofort auf der ganzen Breite des offenen Grabens $(60 \mathrm{~cm})$ ein Quellhorizont, von dem aus die Grube mit sehr kaltem Wasser beliefert wurde. Die Grabenwand fühlte sich kalt an. Beim Tiefergraben stieß ich auf hartes Material. Ein losgebrochenes Handstück des Grubenbodens erwies sich als gefroren (Abb. 13): 3-5 mm mächtige Lamellen aus klarem, sehr hartem Eis trennten hartgefrorene Feinerdeschichten. Haselnußgroße eckige Steinchen waren fest an die Eislamellen angefroren. Das Handstück war sehr kompakt und konnte nur mit dem Spaten zerschlagen werden. Ober- und Unterflächen besaßen - mit der Lupe betrachtet - ein feines Höckerrelief.

Den Frostboden konnte ich bis in eine Tiefe von $65 \mathrm{~cm}$ freilegen, seine absolute Mächtigkeit dagegen nicht bestimmen, da ein Weitergraben mit dem Spaten durch die zunehmende Härte verunmöglicht wurde. Die Eisplatten lagen parallel zur Oberfläche und zogen durch die ganze Feinerdesäule und unter den Steinrahmen durch. Gelegentlich gingen 2 vertikal übereinanderliegende Eisplatten ineinander über. Der Frostboden begann plötzlich, er war scharf gegen die über ihm liegende Fließerde abgegrenzt. Frostboden konnte ich am 10. und 11. August 1955 noch auf Fenga Pitschna feststellen.

Mit diesen Funden ist für die Alpen jahreszeitlicher Frostboden in Zusammenhang mit Strukturboden nachgewiesen. Ich glaube mich zur Annahme berechtigt, daß es sich dabei sogar um Dauerfrostboden handelt (Zeitpunkt der Auffindung, Klima, Mächtigkeit der Vorkommen, Zahl der Eistage im Boden vgl. dazu S. 199.).

In Eurasien folgt die Südgrenze des Dauerfrostbodens i. a. der $-2^{\circ}$ Jahresisotherme. Schostakowitsch (Lit. 10, S. 645) erklärt die Verbreitung der ewigen Gefrornis mit den heutigen Klimabedingungen nach der Formel

$$
\frac{\text { mittlere Lufttemperatur der Monate Dezember-Februar }}{\text { mittlere Schneehöhe im Januar }(\mathrm{cm})} \leq 0,5 \text {. }
$$




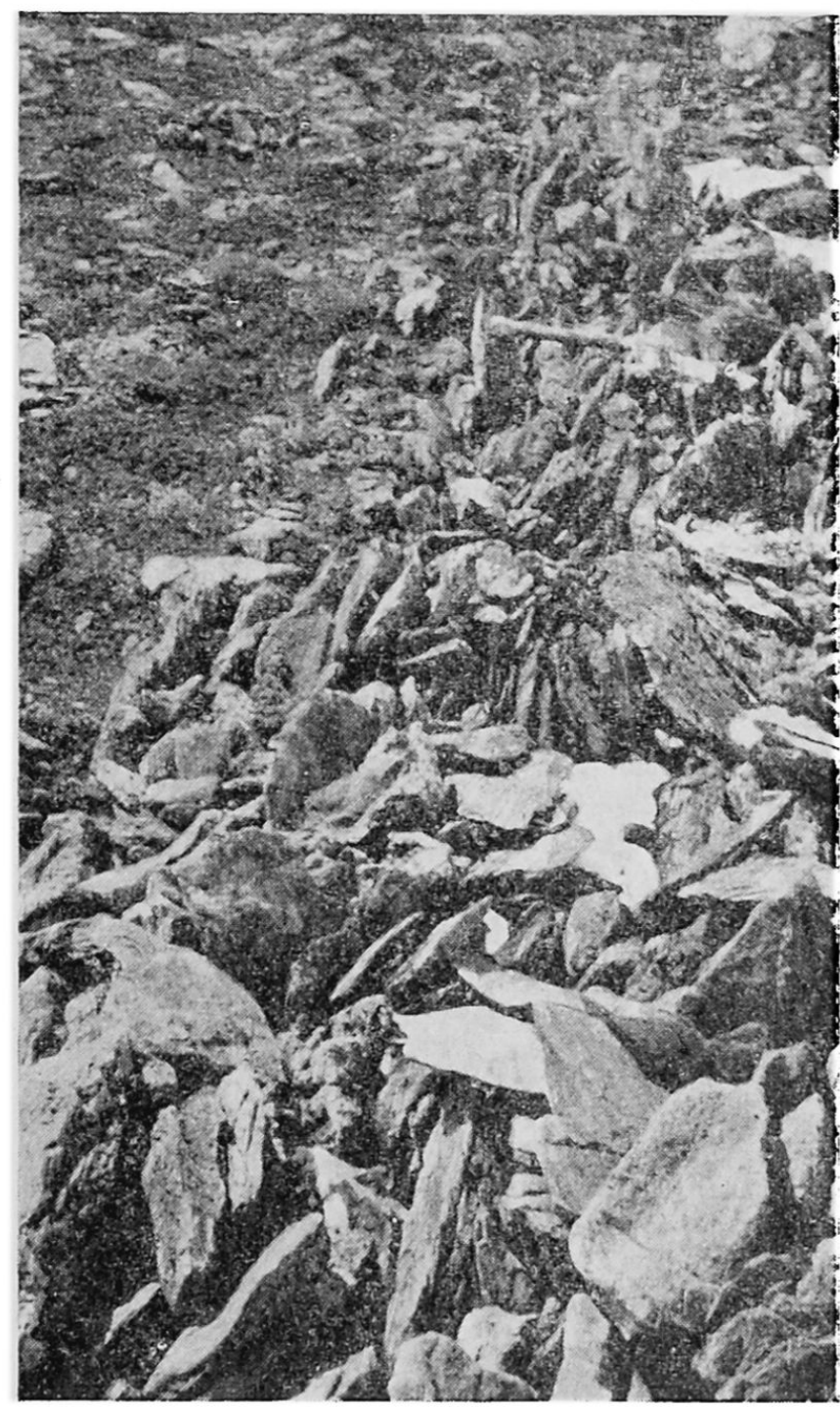

Abb. 19 Ausschnitt aus einem Steinstreifen. - Länge des Spatens $50 \mathrm{~cm}$. 40 bis $50 \mathrm{~cm}$ tiefe Steinfüllung. (Fimberpaß östlich Pt. 2608, 10. 8. 1955.)

Nach Aussagen der Grenzwachtsoldaten, die den Fimberpaß - von dem das Profil in Abb. 10 stammt - periodisch überschreiten, ist dort im Winter der Schnee weggeblasen. Deshalb kann man das beschriebene Vorkommen nach der obigen Formel erklären. - In der Schweiz ist man im subnivalen Bereich anläßlich von Lawinenverbauungen - besonders in Schutthalden - schon öfters auf Dauerfrostboden gestoßen. Dieser bildet sich auch bei wesentlich größeren Schneemächtigkeiten, als die Formel von Schostakowitsch erlauben würde (freundliche Mitteilung von Herrn Dr. ZiNGG, Lawinenforschungsinstitut Weißfluhjoch).

Vergleiche ich alle mir bekannten Steinpolygone der Alpen, so fallen diejenigen über Dauerfrostboden durch ausgeprägte Materialsortierung und i. a. durch größere Sortierungstiefen auf. Vor allem aber treten größere Formen mit größeren Steinen in den Rahmen auf. Frostboden wirkt als Wasserstauer und Gleithorizont für die über ihm liegende Fließerde. Bei Regelation der Fließerde wirkt er als Widerlager, zudem gefriert dieselbe dann von oben und unten zugleich.

Unter den sechseckförmigen Steinpolygonen fielen einige der Steinnetze bei Pt. 2608 durch ihre ungewöhnliche Größe auf. Bei einem deutlich ausgebildeten maßen die Diagonalen sogar 2,7-3 m. Besonders schön waren die Ecken zu seinen - weniger deutlichen - Nachbarpolygonen entwickelt. Steine, deren größte Durchmesser bis $30 \mathrm{~cm}$ maßen, lagen kantengestellt im Rahmen. Die Feinerdesäule trug eine lose Bedeckung aus flachen und kleinen Steinen.

\section{GROSSFORMEN VON STEINSTREIFEN (Abb. 17-19)}

Schon bei schwacher Hangneigung treten keine regelmäßigen Polygonformen mehr auf. Steinnetze und einzelne Steinkreise werden durch die Hangsolifluktion zu länglichen Figuren deformiert (vgl. Abb. 14). Die der Ebene zunächst gelegenen Steinpolygone sind parallel zur Fallinie gestreckt, etwas weiter hangabwärts treten deutlich in die Länge gezogene Steinellipsen auf, die bei zunehmender Hangneigung (10 $20^{\circ}$ ) in lange Steinstreifen übergehen können. Ihr Anfang ist leicht der Beobachtung zugänglich: Die beiden talwärtigen Seiten eines Steinpolygons bilden einen spitzen Winkel, von welchem dann der Steinstreifen ausgeht. Anfänglich weist derselbe meist nur geringe Tiefen auf (weniger als $10 \mathrm{~cm}$ ) und hebt sich nur undeutlich von der Steindecke der Feinerde ab. Gelegentlich verlaufen diese Steinstreifen schnurgerade und parallel zueinander, in anderen Fällen können zwei benachbarte zusammenlaufen oder 
eine leicht gewundene Linie beschreiben. Die Abstände von Steinstreifen zu Steinstreifen sind häufig unregelmäßig, sie variieren zwischen $50-300 \mathrm{~cm}$.

An den talwärts gelegenen Enden gehen die Steinstreifen oft in Steinzungen über, deren Fließstruktur deutlich sichtbar ist: Auf dem Zungenrücken sind die kantengestellten Steine parallel zur Zungenform in Wülsten zusammengestaut. Noch im Spätsommer liegt oft Schnee und Schmelzwasser zwischen diesen Wülsten.

Die Feinerde zwischen den Steinstreifen kann steinarm oder von einer dünnen Steindecke bedeckt sein. Nach der Schneeschmelze sind die Feinerdestreifen stark durchtränkt, oft sinkt man in dieser Fließerde rasch $10-20 \mathrm{~cm}$ tief ein. Vegetation trifft man eher an den Steinstreifen als auf der Feinerde an.

Das Profil durch einen Steinstreifen von Fenga Pitschna in $2650 \mathrm{~m}$ Höhe (Abb. 18) wird durch Abb. 10, rechter Steinrahmenquerschnitt, veranschaulicht. Der Steinrahmen der Polygone über Frostboden im Fimbergebiet ist gleich wie die Steinstreifen ausgebildet. Auffallend ist die Kastenform mit dem breiten Boden, welchen unzählige kleine, kantige Steinchen bedecken. Ende August 1955 floß zwischen den kantengestellten Steinen Wasser talwärts. Der Vorgang der Kantenstellung in den Rahmen läßt sich deutlich beobachten: Beim Gefrieren wölben sich die Feinerdestreifen wie Frostbeulen auf, dabei wirken lateral Kräfte auf die Steinstreifen, in welchen weder Eis noch zusammengefrorene Steine $z u$ finden sind. Steinstreifen treten im Fimbergebiet recht häufig auf; meistens sind sie mehr als $30 \mathrm{~m}$ lang, ihre Breite mißt durchschnittlich $40 \mathrm{~cm}$. Ich fand einen besonders schön ausgebildeten von mehr als $1 \mathrm{~m}$ Breite mit kantengestellten Steinen, deren Durchmesser oft $1 / 2 \mathrm{~m}$ erreichten.

Wie die Steinpolygone, so können auch die Steinstreifen aus präexistierenden Rissen hervorgehen. Der Streifenanfang dieser Formen besitzt als hangwärtige Fortsetzung einen vertikalen $\mathrm{Ri} \beta$ in der Feinerde. Diese Risse lassen sich oft bis unter die Steinfüllung verfolgen (vgl. auch Lit. 4, S. 235 f). Sie messen bis $10 \mathrm{~cm}$ in der Tiefe und $1 \mathrm{~cm}$ in der Breite. Die Steinfüllung gewinnt erst weiter talwärts an Mächtigkeit; der Übergang vom Riß zum Steinstreifen vollzieht sich allmählich. Gelegentlich werden solche Risse durch Schmelzwasser verbreitert und durch Solifluktionsvorgänge mit Steinen der Oberfläche gefüllt. Corbel (Lit. 2) weist darauf hin, daß die Steinstreifen andere Steine besitzen können als die begrenzende Feinerde (gleicher Nachweis in einem Fall im Nationalpark). Wie bei den Steinpolygonen, so findet man auch bei Steinstreifen Formen, bei welchen Feinerde auch in ungefrorenem Zustand weiter hinaufreicht als die Steinfüllung und diese teilweise überdeckt. Auf diese Art vertiefte Steinrahmen und -streifen sind gelegentlich von Vegetation bedeckt.

Höhenlage einiger gut ausgebildeter Frostmusterformen:

Miniaturformen:

Nationalpark

Fimbergebiet

Piz Beverin ( $3000 \mathrm{~m}$ )

Munt della Bescha (2775 m)

Stilfserjoch

Makroformen:

Nationalpark

Fimbergebiet

Davo Lais (NE Piz Lavèr, $2984 \mathrm{~m}$ )

Piz Faschalba (3o48 m) - Grat westwärts bis $\mathrm{Pt}$. 2918

Piz Beverin - Piz Runal (2705 m).

Stilfserjoch

Flühseen NE Juf

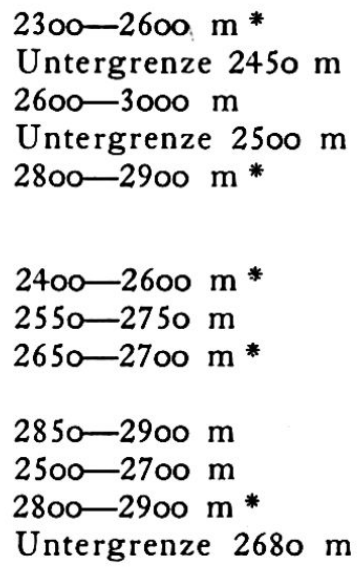

* Höher keine Beobachtungsmöglichkeit. 
Muttensee - Kistenpaß (Streiff-Becker, Geographica 1946)

Silvrettagruppe vor Klostertaler und Larain Ferner. Vorgelände von

Steingletscher

Morteratschgletscher

Glacier de Ferpècle

\author{
ca. $2500 \mathrm{~m}$ \\ $2200-2600 \mathrm{~m}(\mathrm{Kinzl})$ \\ 1960 m (Gelände erst nach 1942 \\ eisfrei) \\ $1950 \mathrm{~m}$ \\ Auf Flächen, die erst 4 Jahre eisfrei \\ noch undeutliche Ausbildung
}

Die meisten Makroformen treten - von solchen auf Moränen und Schotterfeldern weit hinabreichender Gletscher abgesehen - zwischen 2200-2700 m auf. Sie werden im Vorfeld von heutigen Gletschern auf vegetationslosen Flächen gebildet oder fern von Gletschern in der Nähe von Schneeflecken, die das zu ihrer Bildung notwendige Wasser liefern. Auf dem großen und dem kleinen Fimberpaß konnte unter Strukturbodenformen Dauerfrostboden festgestellt werden. Diese Strukturbodenformen bilden sich durch Gefrornis im Winter und Durchtränkungs- und Regelationsfließen im Sommer. Die Materialsortierung reicht so tief, wie die jahreszeitliche Gefrornis wirkt.

Die durch häufige Regelation und Mikrosolifluktion entstehenden regelmäßigen Miniaturformen können hauptsächlich in größeren Höhen nachgewiesen werden (über $2500 \mathrm{~m}$; an Orten mit früher Schneeschmelze, in südexponierten Lagen über $2300 \mathrm{~m}$ ). Entsprechend den kuzfristigen Frösten werden die Bodenpartikel nur bis in $6 \mathrm{~cm}$ Tiefe sortiert.

Zur Hauptfrostwechselzeit in der untern Zone des subnivalen Bereiches (2200$2500 \mathrm{~m}$ ) liegt meist noch Schnee, der das Einwirken kurzfristiger Fröste auf den Boden erschwert. Weil in der oberen Zone des subnivalen Bereiches die Hauptfrostwechselzeit mit der schneefreien Zeit zusammenfällt, finden wir dort die Miniaturformen.

\title{
ZITIERTE LITERATUR
}

1. Allix, A.: Nivation et sols polygonaux dans les Alpes françaises. La Géographie, 39, 1923. 2. CoRBel, J.: Les sols polygonaux: Observations, expériences, genèse. Revue de Géomorphologie dynamique, 2, 1954. - 3. CoRTe, A.: Contribución a la morfologia periglacial especialmente criopedológica de la República Argentina. Societas Geographica Fenniae, 14, 1955. - 4. FurRer, G.: Solifluktionsformen im Schweizerischen Nationalpark. Ergebnisse der wissenschaftlichen Untersuchungen des schweizerischen Nationalparks, IV, H. 29, 1954. - 5. FURRER, G.: "Steingärtchen " in den Alpen. Leben und Umwelt, H. 4, 1955. - 6. FurRer, G: Bodenformen aus dem subnivalen Bereich. Die Alpen, H. 6, 1955. 7. Krumme, O.: Frost und Schnee in ihrer Wirkung auf den Boden im Hochtaunus. Rhein-Mainische Forschungen, H. 13, 1935. - 8. Ruckl., R.: Der Frost im Baugrund. Wien, 1950. - 9. SCHRöDER, P.: Über die vertikale Verteilung der Temperaturschwankungen um den Frostpunkt in Mitteleuropa. Diss. Leipzig, 1912. - 10. TRoLL, C.: Strukturböden, Solifluktion und Frostklimate der Erde. Geolog. Rundschau, H. 7/8, 1944. - 11. WinkLer, E.: Zur Frostverteilung der Schweiz. Mitt. der Geographisch-Ethnographischen Ges. in Zürich, 1937/38. Koordinaten aus der Landeskarte der Schweiz.

\section{LES SOLS POLYGONAUX ET STRIÉS DES ALPES}

Dans les régions alpines, il faut distinguer entre les grandes et petites structures des sols polygonaux. Les petites structures ont comme source les gelées de courte durée mais fréquentes et peuvent être comparées avec celles des hautes montagnes tropicales. Les structures de grande taille résultent des gelées saisonnières comme les sols polygonaux des régions polaires.

En cas de gelées de courte durée, le sol gèle pour quelques heures ou jours jusqu'à la profondeur de 3 à $6 \mathrm{~cm}$, ou bien il se forme du «pipkrake». Ce cas ne se produit que dans des conditions particulières: la température des couches superficielles du sol reste au-dessus de $0^{\circ}$, tandis que l'air proche du sol montre des valeurs négatives.

Les gelées nocturnes forment par leur alternance fréquente (regel) les sols polygonaux (le mouvement produit par une grande brosse de pipkrake se trouvant sur une plaine horizontale est montré dans la fig. 2). D'autres petites formes proviennent de la couche de neige et de la coulée des petites pierres qui restent fixées dans les fissures de dessication.

Sur les pentes, les polygones pierreux se déforment par la solifluction en sols striés.

Pour la première fois dans les Alpes, il était possible de fournir des renseignements sur des polygones au-dessus du «permafrost " dans la région du Passo di Fenga (Val Sinestra, dans le voi- 
sinage de Scuol) à une altitude de $2600 \mathrm{~m}$. Ces structures sont plus grandes que les microformes et se forment beaucoup plus lentement. Une autre sorte de polygones résulte d'un réseau de fissures. Ces fissures effectuent une congélation non proportionnée du sol et ensuite une migration d'un tas de pierres vers les fissures.

Les grandes formes des sols polygonaux se trouvent avant tout entre $2200 \mathrm{~m}$ et $2700 \mathrm{~m}$ d'altitude, les petites structures au-dessus de $2500 \mathrm{~m}$. Ces dernières sont moins fréquentes dans des positions plus basses parce qu'au printemps, les nuits de gel étant très fréquentes, la neige protège le sol de l'action des gelées nocturnes.

\section{PROFIL HYPSOMÉTRIQUE DU FAITE DES ALPES CENTRALES}

\section{MAX BOUËT}

La forme et la hauteur de la chaîne des Alpes jouent assurément un rôle important dans la distribution géographique, la direction et la fréquence du vent, et en particulier du fœhn. On sait en effet que ce dernier n'apparaît pas dans toutes les vallées en même temps ni avec la même intensité, ce dont le relief est certainement en grande partie responsable. De là l'idée d'étudier l'extension verticale de cet obstacle naturel et d'en évaluer l'importance. Contraint de fixer des limites, j'ai déterminé ici l'altitude moyenne des Alpes dans leur partie la plus élevée, située en majeure partie en Suisse ou le long de sa frontière méridionale.

\section{MÉTHODE}

Si l'on envisage les Alpes comme un obstacle aux vents, il est logique de rechercher l'altitude de leur plus haut profil submersible en le considérant sous l'angle de la statique des fluides. Me plaçant à ce point de vue et tenu de simplifier un problème topographique en lui-même compliqué, je me suis limité au faite alpin défini comme la ligne de partage des eaux entre les bassins du Rhône, du Rhin et du Danube d'une part, du Pô et de l'Adige d'autre part. J'ai donc laissé de côté les chaînes et massifs situés en-deça ou au-delà du dit faîte et qui par endroits peuvent pourtant lui être légèrement supérieurs, comme par exemple le groupe de la Jungfrau.

J'ai alors calculé, par sections, l'altitude moyenne du faite alpin entre le col du Petit-Saint-Bernard à la frontière franco-italienne et le passo di Rèsia (Reschenscheideck) à la frontière autro-italienne. La présence du puissant massif du Mont-Blanc où culmine la chaîne alpine justifiait l'extension du profil vers le Sud-Ouest, au-delà des Alpes italo-suisses proprement dites.

Le matériel cartographique utilisé fut le suivant: a) Au 1:50 000: Carte nationale de la Suisse; Carte du Mont-Blanc de Barbey/Imfeld/Kurz; Nouvelle carte de France; Carta delle zone turistiche d'Italia. b) Au 1:100 000: Carte topographique de la Suisse (Dufour); Carta del Istituto geografico militare.

C'est avant tout le premier de ces documents, la très remarquable «Carte nationale de la Suisse» au 1:50000 en cours de publication, qui a servi au pointage des nombreuses cotes d'altitude; les autres ont été consultés pour les parties de la crête situées entièrement en territoire français ou italien.

Après avoir relevé 921 cotes (1,6 par kilomètre) indiquées sur la carte ou déduites des isohypses, je les ai reportées graphiquement sous forme d'une ligne brisée reliant creux et sommets. Les distances horizontales nécessaires au calcul de l'altitude furent mesurées le long de 55 alignements sur lesquels vinrent se projeter les points cotés; ces alignements, longs de $8,6 \mathrm{~km}$. en moyenne, furent choisis de manière à s'adapter le mieux possible au tracé parfois très sinueux de la ligne de partage des eaux. Cette mesure des distances par projection est simple et précise; elle substitue à la coupe verticale réelle un profil contracté dans le sens horizontal sans toutefois modifier l'alti- 